\title{
Preparation of L-vancosamine-related glycosyl donors
}

\author{
Kei Kitamura ${ }^{1}$, Masayuki Shigeta ${ }^{1}$, Yoshihiko Maezawa ${ }^{1}$, Yukie Watanabe ${ }^{1}$, Day-Shin Hsu ${ }^{1,3}$, Yoshio Ando ${ }^{1}$, \\ Takashi Matsumoto ${ }^{2}$ and Keisuke Suzuki ${ }^{1}$
}

An improved practical synthesis of L-vancosamine-related glycosyl donors is described. The key steps include (1) stereoselective addition of methylcerium reagent to oximino ether and (2) stereoselective hydrogenation of exocyclic unsaturated glycoside in the presence of Wilkinson catalyst with $\mathrm{C}(5)$ inversion to give L-vancosamine derivatives. Three glycosyl donors were prepared, and their reactivities in the aryl $C$-glycoside formation were compared. Conversion of primary amine and azide to the corresponding $\mathbf{N}, \mathbf{N}$-dimethyl derivative is also described.

The Journal of Antibiotics (2013) 66, 131-139; doi:10.1038/ja.2013.2; published online 20 February 2013

Keywords: glycosyl donor; L-vancosamine; practical synthesis

\section{INTRODUCTION}

L-Vancosamine and its $N, N$-dimethyl derivative are found as the sugar constituents of several antibiotics. For example, L-vancosamine ${ }^{1,2}$ is included in vancomycin, a glycopeptide antibiotic that is important for the treatment of methicillin-resistant Staphylococcus aureus. ${ }^{3}$ The $\mathrm{N}, \mathrm{N}$-dimethyl analog occurs as an $\mathrm{O}$-glycoside in the nocardicyclin antibiotics $^{4,5}$ and as a $C$-glycoside in the pluramycin-hedamycin class antibiotics (Figure 1). ${ }^{6-8}$

Many methods have been recorded for synthesizing the vancosamine derivatives starting either from carbohydrates ${ }^{9-16}$ or noncarbohydrates. ${ }^{17-28}$ However, most of the methods had problems on practicality; for example, use of toxic reagents or expensive starting materials. Herein, we record a viable method for preparing glycosyl donors of L-vancosamine derivatives.

\section{RESULTS AND DISCUSSION}

The route is primarily based on the report by Thang et al., ${ }^{29}$ which has been improved ${ }^{30}$ in terms of the stereoselectivity, and thus overall efficiency. The starting material was a known ketone $\mathbf{1}^{31}$ derived from commercially available methyl $\alpha$-D-mannopyranoside, treatment of which with $O$-methyl hydroxylamine hydrochloride

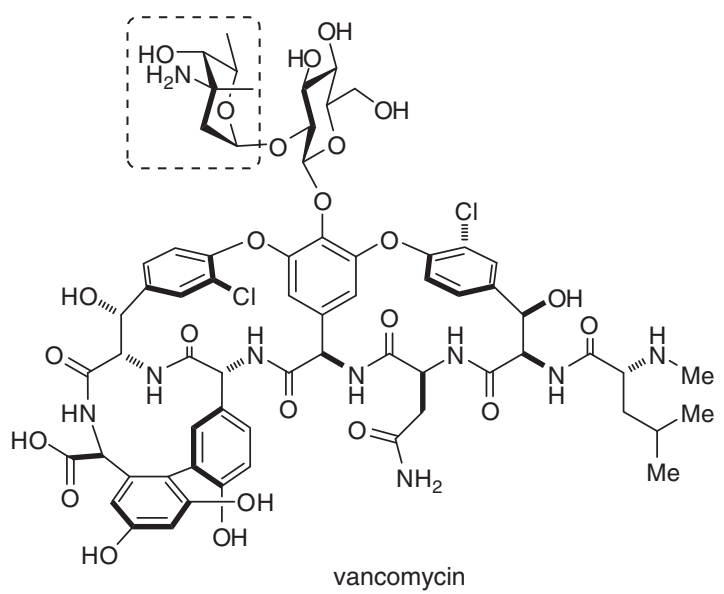

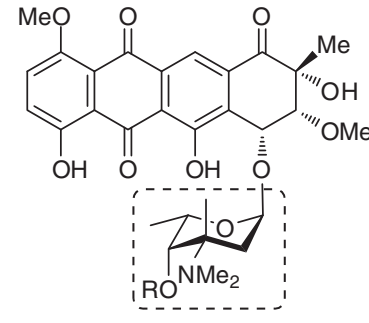

nocardicyclins

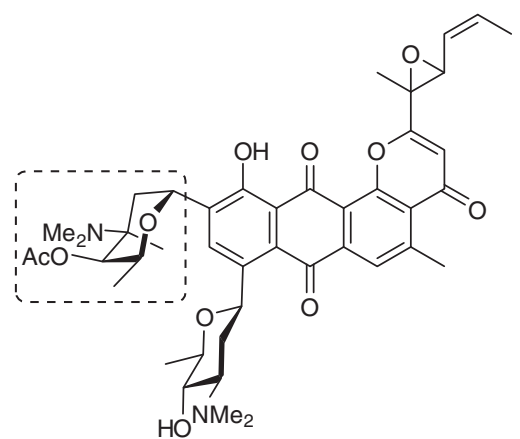

pluramycin A

Figure 1 L-Vancosamine in antibiotic structures.

\footnotetext{
${ }^{1}$ Department of Chemistry, Tokyo Institute of Technology, Tokyo, Japan and ${ }^{2}$ School of Pharmacy, Tokyo University of Pharmacy and Life Sciences, Tokyo, Japan ${ }^{3}$ Current address: Department of Chemistry and Biochemistry, National Chung Cheng University, Chiayi 62102, Taiwan.

Correspondence: Professor T Matsumoto, School of Pharmacy, Tokyo University of Pharmacy and Life Sciences, 1432-1, Horinouchi, Hachioji, Tokyo 192-0392, Japan. E-mail: tmatsumo@toyaku.ac.jp

or Professor K Suzuki, Department of Chemistry, Tokyo Institute of Technology, 2-12-1, O-okayama, Meguro-ku, Tokyo 152-8551, Japan.

E-mail: ksuzuki@chem.titech.ac.jp

Dedicated to Professor Kuniaki Tatsuta for his great synthetic expeditions, having conquered 101 peaks, including the 4 major antibiotics.

Received 4 December 2012; revised 25 December 2012; accepted 30 December 2012; published online 20 February 2013
} 
( $\mathrm{NaOAc}, \mathrm{MeOH}$, room temperature, 6h) gave oximino ether 2 in $86 \%$ yield. Treatment of $\mathbf{2}$ with the methylcerium species, ${ }^{32,33}$ generated by mixing $\mathrm{MeLi}$ and anhydrous $\mathrm{CeCl}_{3}$ (tetrahydrofuran (THF), $-78^{\circ} \mathrm{C} \rightarrow 0^{\circ} \mathrm{C}, 4 \mathrm{~h}$ ), gave amine 3 as a single product. The stereostructure of $\mathbf{3}$ was assigned by the NOE experiment as shown in Figure 2. After conversion of amine 3 to trifluoroacetamide $4\left[\left(\mathrm{CF}_{3} \mathrm{CO}\right)_{2} \mathrm{O}\right.$, pyridine, 4-(dimethylamino)pyridine (DMAP), $\left.\mathrm{CH}_{2} \mathrm{Cl}_{2}, 30 \mathrm{~min}\right]$, the $\mathrm{N}-\mathrm{O}$ bond was cleaved with samarium iodide ${ }^{34,35}\left(\mathrm{MeOH}\right.$, THF, $\left.0{ }^{\circ} \mathrm{C}, 30 \mathrm{~min}\right)$, giving amide 5 in $95 \%$ yield. Recrystallization (EtOAc, hexane) gave nice single crystals of $\mathbf{5}$

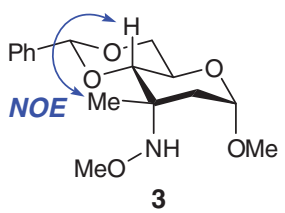

Figure 2 NOE study of amine 3 .

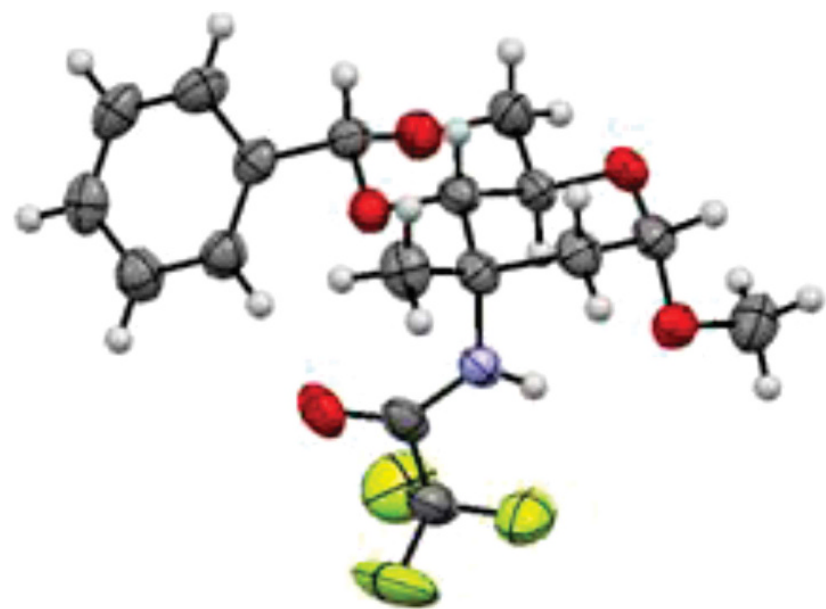

Figure $3 \mathrm{X}$-ray structure of trifluoroacetamide $\mathbf{5}$.
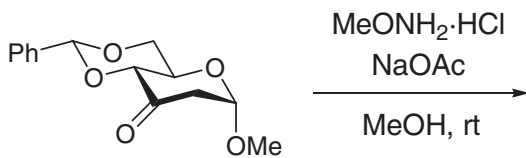

$86 \%$

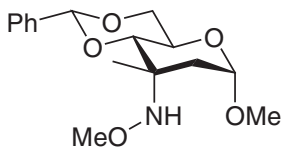

$\left(\mathrm{CF}_{3} \mathrm{CO}\right)_{2} \mathrm{O}$ pyridine, DMAP

$\mathrm{CH}_{2} \mathrm{Cl}_{2}, 0^{\circ} \mathrm{C}$

3

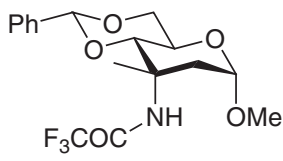

5

Scheme 1 Preparation of trifluoroacetamide 5.

$95 \%$ (2 steps) suitable for X-ray analysis, confirming the stereochemical identity (Figure 3 and Scheme 1).

The benzylidene acetal in $\mathbf{5}$ was cleaved with $\mathrm{N}$-bromosuccinimide ${ }^{36,37}$ (pyridine, $\mathrm{CCl}_{4}$, reflux, $4 \mathrm{~h}$ ) to give bromide 6 in $87 \%$ yield. Note that this process should be conducted at low concentration $(<30 \mathrm{~mm})$ : When performed at higher concentration the yield was not reproducible, and unidentified products were produced presumably by the competing bromination of the trifluoroacetamide in $5 .{ }^{38}$ Recrystallization $\left(\mathrm{Et}_{2} \mathrm{O}\right.$, hexane) gave nice single crystals of $\mathbf{6}$ suitable for X-ray analysis (Figure 4), confirming that the benzylidene acetal in $\mathbf{5}$ was

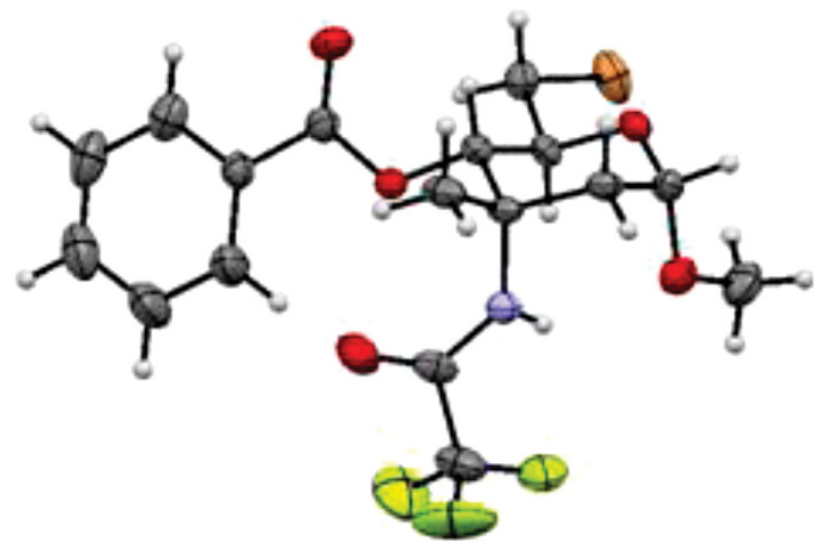

Figure $4 \mathrm{X}$-ray structure of bromide 6 .

Table 1 Hydrogenation of enol ether 7

\begin{tabular}{|c|c|c|c|}
\hline Run & Conditions & Yield of $\mathbf{8} / \%$ & Yield of epi-8/\% \\
\hline 1 & $\mathrm{H}_{2}$ (1 atm), $10 \% \mathrm{Pd} / \mathrm{C}, \mathrm{MeOH}, \mathrm{rt}$ & 67 & 29 \\
\hline 2 & $\mathrm{H}_{2}$ (1 atm), Raney $\mathrm{Ni}$, EtOH, rt & - & - \\
\hline 3 & $\mathrm{H}_{2}$ (1 atm), $\mathrm{Pd}(\mathrm{OH})_{2}, \mathrm{MeOH}, \mathrm{rt}$ & 75 & 22 \\
\hline 4 & $\mathrm{Et}_{3} \mathrm{SiH}, \mathrm{AgBF}_{4}, \mathrm{CH}_{2} \mathrm{Cl}_{2}, \mathrm{rt}$ & - & - \\
\hline 5 & $\mathrm{H}_{2}$ (1 atm), $\mathrm{RhCl}\left(\mathrm{PPh}_{3}\right)_{3}$, toluene, EtOH, rt & 96 & 3 \\
\hline
\end{tabular}
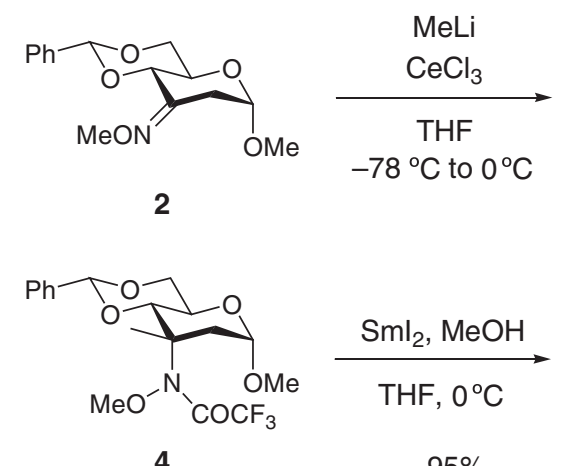

4

$95 \%$ 

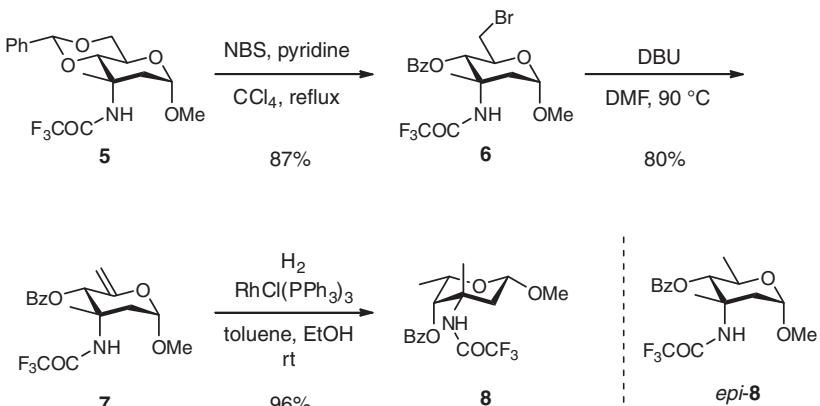

Scheme 2 Preparation of benzoate $\mathbf{8}$.

regioselectively cleaved. Dehydrobromination of $\mathbf{6}$ was effected by using 1,8-diazabicyclo[5.4.0] undec-7-ene (DBU) ( $N, N$-dimethylformamide (DMF), $90{ }^{\circ} \mathrm{C}, 4 \mathrm{~h}$ ) to give enol ether 7 in $80 \%$ yield, ${ }^{39}$ which was subjected to catalytic hydrogenation of the exocyclic 5,6-double bond.

Our previous protocol ${ }^{30}$ using $10 \% \mathrm{Pd} / \mathrm{C}(\mathrm{MeOH}$, room temperature, $12 \mathrm{~h})$ suffered from the low stereoselectivety $(<7: 3)$. The main product 8 was produced in $67 \%$ yield, and the C(5)-epimer, epi-8 in $29 \%$ yield (Table 1 , run 1 ). Although these stereoisomers ( 8 and epi-8) were easily separable by silica-gel chromatography, we sought for a better protocol to improve the stereoselectivity and the yield. In spite of the originally reported protocol, ${ }^{29}$ use of Raney $\mathrm{Ni}$ as the catalyst gave no hydrogenated products in our hands (run 2). Pearlman's catalyst $\left(\mathrm{Pd}(\mathrm{OH})_{2} / \mathrm{C}\right)$ gave products $\mathbf{8}$ and epi-8 in a better stereoselectivity (run 3 ). On the other hand, the combination of triethylsilane with Lewis acids gave no desired compound (run 4). Finally, we found that the hydrogenation of 7 over Wilkinson catalyst $^{40}(5 \mathrm{~mol} \%$, toluene, EtOH, room temperature, $10 \mathrm{~h})$ proceeded in an excellent stereoselectively to give the desired stereoisomer 8 in $96 \%$ yield (run 5; Scheme 2).

Having the key intermediate $\mathbf{8}$ in hand, we prepared three vancosaminyl acetate donors $\mathbf{9}, 12$ and $\mathbf{1 5}$, differing in the protection of 3-amino and 4-hydroxy groups (Figure 5). Preparation of 4-Obenzoyl donor 9 was previously described, ${ }^{30}$ and the routes to amide acetate 12 and azide $\mathbf{1 5}$ are outlined in Schemes 3 and 4, respectively.

Scheme 3 shows preparation of amide acetate 12. The 4-O-benzoyl group in 8 was selectively removed by treatment with $\mathrm{Mg}(\mathrm{OMe})_{2}$ $\left(\mathrm{MeOH}, 0{ }^{\circ} \mathrm{C}, 1.5 \mathrm{~h}\right)$ to give the corresponding alcohol $10,{ }^{41}$ which was protected with a benzyl group $(\mathrm{BnBr}, \mathrm{NaH}, \mathrm{DMF}$, room temperature, $2 \mathrm{~h}$ ) to give benzyl ether 11 in $80 \%$ yield (two steps). Hydrolysis of 11 in $20 \%$ aqueous $\mathrm{AcOH}\left(100{ }^{\circ} \mathrm{C}, 3.5 \mathrm{~h}\right)^{42}$ followed by acetylation $\left(\mathrm{Ac}_{2} \mathrm{O}, \mathrm{DMAP}\right.$, pyridine, room temperature, $\left.11 \mathrm{~h}\right)$ gave glycosyl acetate $\mathbf{1 2}$ in $92 \%$ yield (two steps).

In our synthetic study on the pluramycin-class antibiotics, we needed the glycosyl donors with different protection for the $\mathrm{C}(3)$ amino group. One of the promising donors was azide 15. After hydrolysis of 11 by $5 \mathrm{M}$ aqueous $\mathrm{NaOH}\left(\mathrm{MeOH}, 40^{\circ} \mathrm{C}, 9 \mathrm{~h}\right)$, the resulting free amine $\mathbf{1 3}$ was subjected to the diazo-transfer reaction by treatment of $\mathrm{TeN}_{3}{ }^{43-45}$ in the presence of $\mathrm{CuSO}_{4}\left(\mathrm{MeOH}, \mathrm{H}_{2} \mathrm{O}\right.$, room temperature, $2.5 \mathrm{~h}$ ), giving the desired azide $\mathbf{1 4}$ in $95 \%$ yield (two steps). Recently, Kitamura et $a l^{45}$ have developed diazo-transfer reagent 16, which is stable against heat and impact. Indeed, use of 16 for the same conversion $(13 \rightarrow 14)$ proceeded smoothly (DMAP, $\mathrm{MeCN}$, room temperature, $1 \mathrm{~h})$, giving azide $\mathbf{1 4}$ in $91 \%$ yield. Recrystallization (hexane, EtOAc) gave nice single crystals of $\mathbf{1 4}$ amenable for the X-ray analysis (Figure 6). Hydrolysis of $14(20 \%$
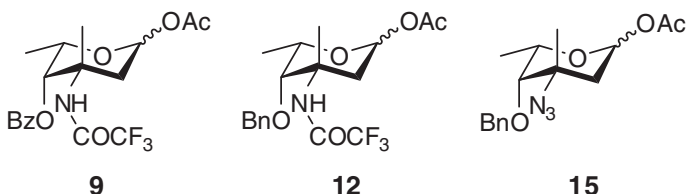

Figure 5 Glycosyl donors 9,12 and 15.

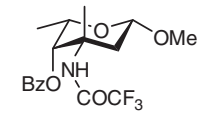

8

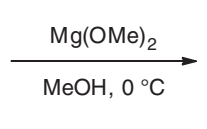

$83 \%$

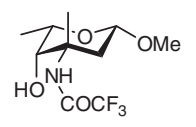

10

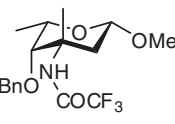

11

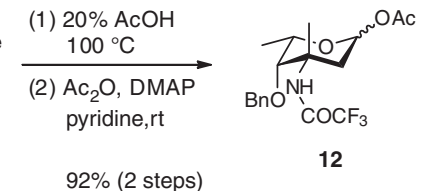

$92 \%$ (2 steps)

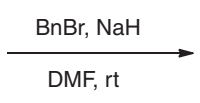

$91 \%$
Scheme 3 Preparation of amide acetate 12.
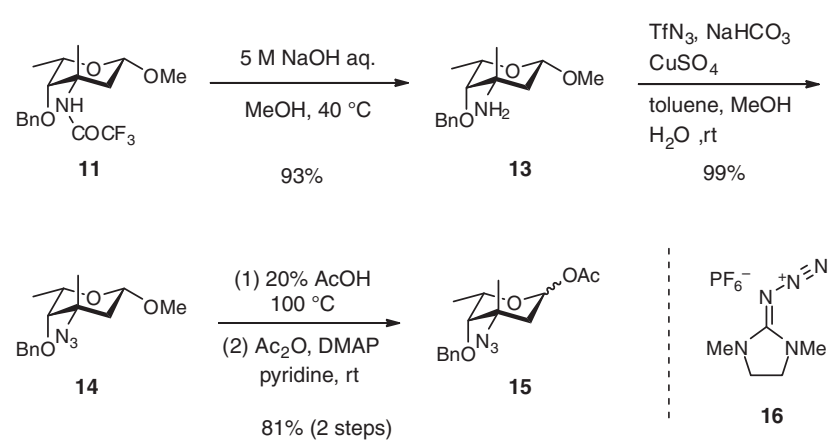

Scheme 4 Preparation of azide acetate 15.

aqueous $\left.\mathrm{AcOH}, 100^{\circ} \mathrm{C}, 3.5 \mathrm{~h}\right)$ followed by acetylation $\left(\mathrm{Ac}_{2} \mathrm{O}, \mathrm{DMAP}\right.$, pyridine, room temperature, $11 \mathrm{~h}$ ) gave glycosyl actetate 15 in $81 \%$ yield (two steps).

With three glycosyl donors $\mathbf{9 , 1 2}$ and $\mathbf{1 5}$ in hand, their reactivities were assessed by the aryl $C$-glycosidation with phenol 17 in the presence of $25 \mathrm{~mol} \%$ of $\mathrm{Sc}(\mathrm{OTf})_{3}$ (Drierite, 1,2-dichloroethane). In the case of C(3)-trifluoroacetamide-substituted acetates 9 and 12, Cglycosides 18 and 19 were obtained in $94 \%$ and $88 \%$ yield, respectively (runs 1 and 2). However, the azide acetate $\mathbf{1 5}$ gave poor result, giving $C$-glycoside 20 in $48 \%$ yield (run 3; Table 2).

Finally described is a reliable protocol for converting these amino and azido compounds into the $\mathrm{N}, \mathrm{N}$-dimethyl derivatives. Primary amine 13 was converted to the dimethylamino sugar 21 in excellent yield by treatment with formalin and sodium cyanoborohydride $\left(\mathrm{MeCN}, 0{ }^{\circ} \mathrm{C}, 15 \mathrm{~min}\right)$. Azide 14 could also be converted in one pot to $\mathrm{N}, \mathrm{N}$-dimethylamine 21 ( $74 \%$ yield) by a modified protocol of our previous report ${ }^{46,47}\left(\mathrm{PMe}_{3}, \mathrm{CH}_{2} \mathrm{Cl}_{2}\right.$, room temperature, $4 \mathrm{~h}$; aq $\mathrm{HCHO}, \mathrm{NaBH}_{3} \mathrm{CN}, \mathrm{AcOH}, \mathrm{MeCN}$, room temperature, $1 \mathrm{~h}$; Scheme 5).

In conclusion, an improved synthesis of L-vancosamine donors has been described. Conversion of primary amine and azide to the corresponding $\mathrm{N}, \mathrm{N}$-dimethyl derivative is also described. 

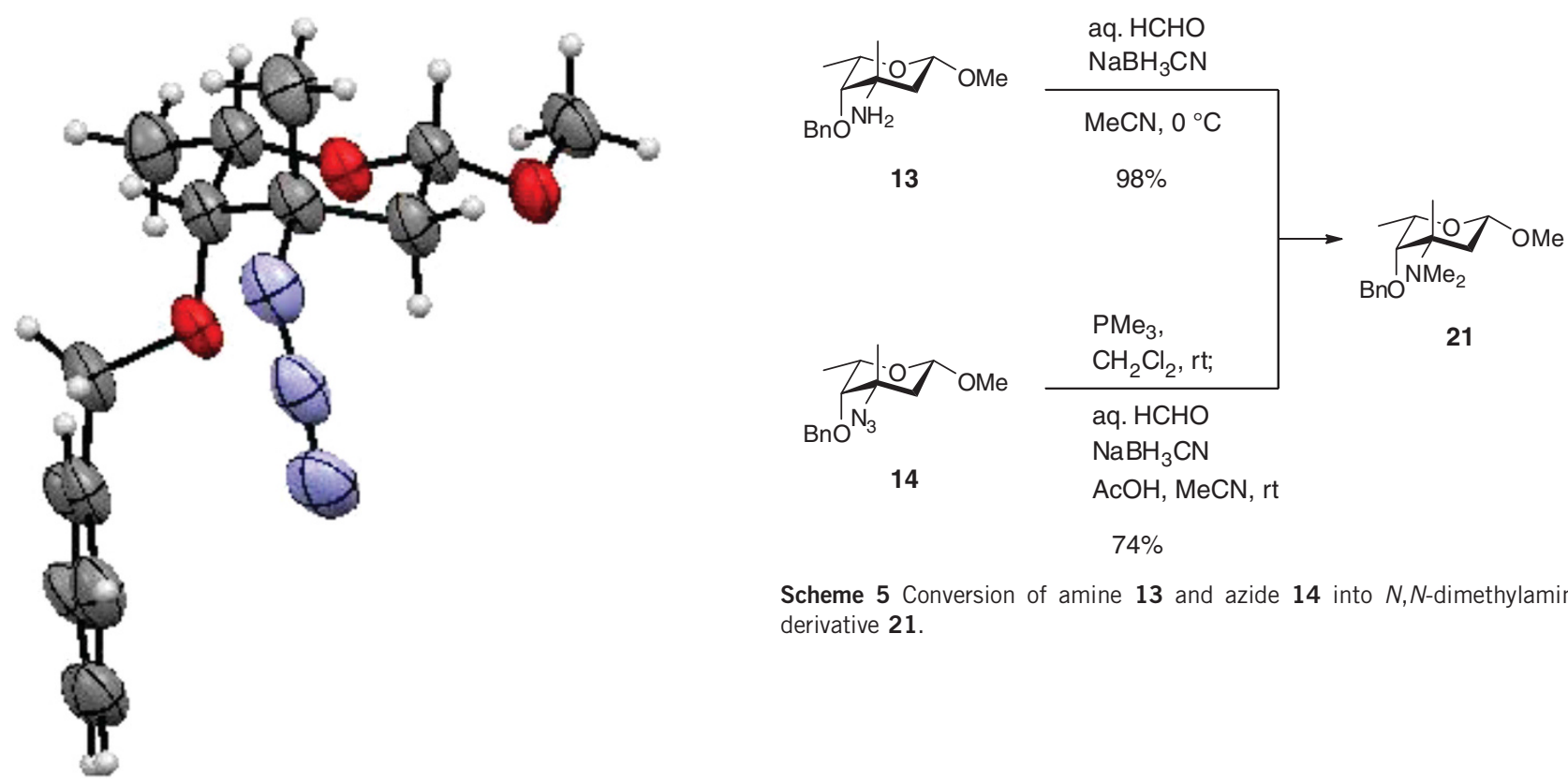

Scheme 5 Conversion of amine 13 and azide 14 into $\mathrm{N}, \mathrm{N}$-dimethylamino derivative 21 .

Figure 6 X-ray structure of azide 14.
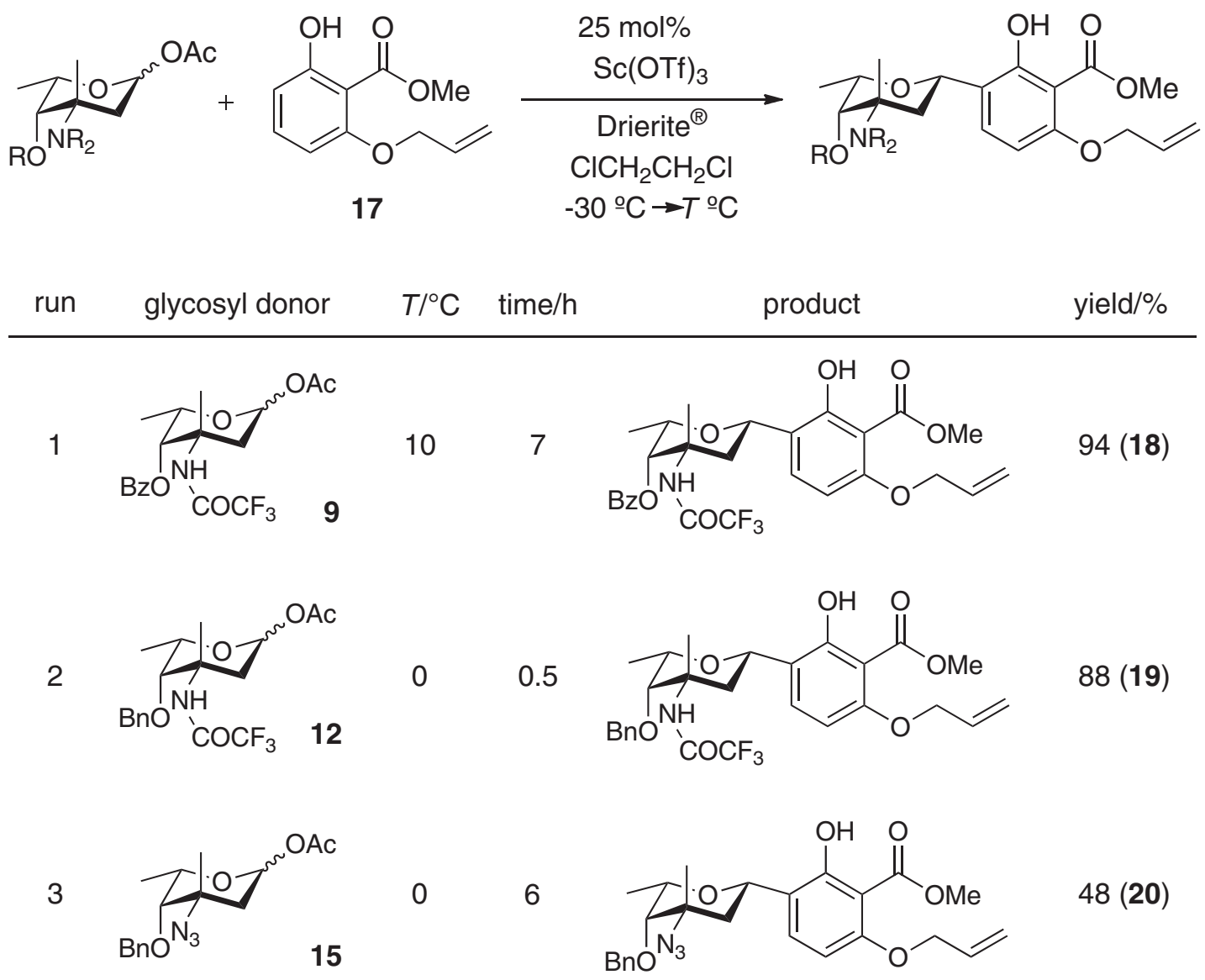


\section{EXPERIMENTAL PROCEDURE}

General

All experiments dealing with air- and moisture-sensitive compounds were conducted under an atmosphere of dry argon. Ethereal solvents (anhydrous; Kanto Chemical Co., Inc., Tokyo, Japan) were used as received. Dichloromethane and 1,2-dichloroethane were distilled successively from $\mathrm{P}_{2} \mathrm{O}_{5}$ and $\mathrm{CaH}_{2}$, and stored over 4A molecular sieves. For TLC analysis, Merck precoated plates (Merck, Darmstadt, Gemany) (silica gel 60 F254, Art 5715, $0.25 \mathrm{~mm}$ ) were used. For flash column chromatography, silica gel $60 \mathrm{~N}$ (Spherical, neutral, 23-210 $\mu \mathrm{m}$ ) from Kanto Chemical was used. Preparative TLC was performed on Merck silica gel 60 PF254 (Art 7747). Determinations of m.p.were performed by using a Yanako MP-500 (Yanako, Kyoto, Japan) instrument. ${ }^{1} \mathrm{H}$ NMR and ${ }^{13} \mathrm{C}$ NMR spectra were measured on a JEOL JNM ECX-500 (JEOL, Tokyo, Japan) (500 MHz), a JEOL JNM AL-400 (400 MHz), a JEOL JNM Lambda-400 $(400 \mathrm{MHz})$ or a JEOL JNM AL-300 $(300 \mathrm{MHz})$ spectrometer, and are reported in p.p.m. using tetramethysilane as an internal standard (tetramethysilane $=0$ p.p.m.). ${ }^{1} \mathrm{H}$ NMR spectra data are reported as: $(\delta$ shift $)((s=$ singlet, $\mathrm{d}=$ doublet, $\mathrm{t}=$ triplet, $\mathrm{q}=$ quartet, quin $=$ quintet, $\mathrm{m}=$ multiplet, $\mathrm{br}=$ broad), (integration) and $(J=$ coupling constant in $\mathrm{Hz})$ ). IR spectra were recorded on a Jasco IR-Report 100 (Jasco, Tokyo, Japan) or a Perkin Elmer Spectrum 100 FTIR spectrometer (Perkin Elmer, Waltham, MA, USA). Attenuated total reflectance (ATR) FTIR spectra were recorded on a Perkin Elmer 100 spectrometer. Optical rotations $\left([\alpha]_{D}\right)$ were measured on a Jasco DIP-1000 polarimeter. Elemental analyses were recorded on an Elementar vario MICRO cube analyzer (Elementar, Tokyo, Japan).

\section{$O$-Methyl methyl 4,6-O-benzylidene-2,3-dideoxy- $\alpha$-D-erythro- hexopyranosid-3-ulose oxime (2)}

A solution of ketone $1(52.0 \mathrm{~g}, 197 \mathrm{mmol}), O$-methylhydroxylamine hydrochloride $(29 \mathrm{~g}, 347 \mathrm{mmol})$ and $\mathrm{NaOAc}(29.3 \mathrm{~g}, 358 \mathrm{mmol})$ in $\mathrm{MeOH}(1040 \mathrm{ml})$ was stirred at room temperature for $6 \mathrm{~h}$. After cooling to $0{ }^{\circ} \mathrm{C}$, the mixture was poured into water $(2 \mathrm{l})$. The precipitates were collected by filtration to provide O-methyl oxime $2(49.5 \mathrm{~g}, 86 \%)$ as white solids, which were used in the next step without further purification. An analytical sample of 2 was obtained by recrystallization from $\mathrm{MeOH}$ as colorless needles.

$\mathrm{R}_{f} 0.40$ (hexane/EtOAc $\left.=7 / 3\right) ;$ m.p. $209-210{ }^{\circ} \mathrm{C}(\mathrm{MeOH}) ;[\alpha]_{\mathrm{D}}^{30}+173(c$ 1.03, $\left.\mathrm{CHCl}_{3}\right) ;{ }^{1} \mathrm{H}$ NMR $\left(\mathrm{CDCl}_{3}, 400 \mathrm{MHz}\right): 2.24(\mathrm{dd}, 1 \mathrm{H}, J=4.6,15.4 \mathrm{~Hz})$, $3.37(\mathrm{~s}, 3 \mathrm{H}), 3.52(\mathrm{dd}, 1 \mathrm{H}, J=1.0,15.4 \mathrm{~Hz}), 3.83(\mathrm{dd}, 1 \mathrm{H}, J=10.0,10.5 \mathrm{~Hz})$, $3.92(\mathrm{~s}, 3 \mathrm{H}), 4.05(\mathrm{ddd}, 1 \mathrm{H}, J=4.9,9.8,10.0 \mathrm{~Hz}), 4.24(\mathrm{~d}, 1 \mathrm{H}, J=9.8 \mathrm{~Hz}), 4.31$ (dd, $1 \mathrm{H}, J=4.9,10.5 \mathrm{~Hz}$ ), 4.90 (brd, $1 \mathrm{H}, J=4.6 \mathrm{~Hz}$ ), $5.63(\mathrm{~s}, 1 \mathrm{H}), 7.34-7.39$ $(\mathrm{m}, 3 \mathrm{H}), 7.51-7.55(\mathrm{~m}, 2 \mathrm{H}) ;{ }^{13} \mathrm{C} \mathrm{NMR}\left(\mathrm{CDCl}_{3}, 100 \mathrm{MHz}\right): 30.8,54.8,61.9$, 64.7, 69.4, 78.1, 98.4, 102.4, 126.5, 128.2, 129.1, 137.0, 148.8; IR (ATR): 3059, 2943, 2866, 1643,1367, 1124, 1049, 991, 748, $698 \mathrm{~cm}^{-1}$. Anal. calcd for $\mathrm{C}_{15} \mathrm{H}_{19} \mathrm{NO}_{5}$ : C, 61.42; H, 6.53. Found: C, 61.72; H, 6.77.

\section{Methyl 4,6-O-benzylidene-2,3-dideoxy-3-C-methyl-3-} (methoxyamino)- $\alpha$-D-ribo-hexopyranoside (3)

Cerium chloride heptahydrate $(36.8 \mathrm{~g}, 98.8 \mathrm{mmol})$ was dried under vacuum $(0.1 \mathrm{~mm} \mathrm{Hg})$ by the Imamoto procedure. ${ }^{33}$ The resulting powders were cooled under vacuum, and the flask was flushed with argon. Dry THF $(400 \mathrm{ml})$ was added, and the resulting suspension was stirred vigorously at room temperature for $12 \mathrm{~h}$. The mixture was cooled at $-78{ }^{\circ} \mathrm{C}$, to which $\mathrm{MeLi}\left(1.09 \mathrm{M}\right.$ in $\left.\mathrm{Et}_{2} \mathrm{O}, 90 \mathrm{ml}, 98.1 \mathrm{mmol}\right)$ was added dropwise. The yellow suspension was stirred for $1 \mathrm{~h}$, and a solution of $O$-methyl oxime $2(10.1 \mathrm{~g}, 34.4 \mathrm{mmol})$ in THF $(100 \mathrm{ml})$ was added dropwise. After $1 \mathrm{~h}$ at $-78^{\circ} \mathrm{C}$, the reaction mixture was gradually warmed to $0{ }^{\circ} \mathrm{C}$, and the stirring was continued for $3 \mathrm{~h}$. To the resulting brown suspension saturated aqueous $\mathrm{NH}_{4} \mathrm{Cl}$ was added, and the products were extracted with EtOAc $(3 \times)$. The combined organic extracts were successively washed with brine, saturated aqueous $\mathrm{NaHCO}_{3}$ and brine, and then dried $\left(\mathrm{Na}_{2} \mathrm{SO}_{4}\right)$. Filtration and concentration in vacuo gave amine 3 (11.4 g) as light brown oil, which was used in the next step without further purification. An analytical sample of $\mathbf{3}$ was obtained by column chromatography (hexane/EtOAc $=7 / 3$ ) and recrystallization from $\mathrm{Et}_{2} \mathrm{O} /$ hexane.
$\mathrm{R}_{f} 0.53$ (hexane/EtOAc $\left.=7 / 3\right) ;$ m.p. $87-88^{\circ} \mathrm{C}\left(\mathrm{Et}_{2} \mathrm{O} /\right.$ hexane); $[\alpha]_{D}^{30}+105(c$ 1.02, $\left.\mathrm{CHCl}_{3}\right) ;{ }^{1} \mathrm{H}$ NMR $\left(\mathrm{CDCl}_{3}, 400 \mathrm{MHz}\right): 1.29$ (s, 3H), 1.57 (dd, $1 \mathrm{H}, J=4.6$, $14.9 \mathrm{~Hz}), 2.36(\mathrm{dd}, 1 \mathrm{H}, J=0.6,14.9 \mathrm{~Hz}), 3.38(\mathrm{~s}, 3 \mathrm{H}), 3.49(\mathrm{~d}, 1 \mathrm{H}, J=9.8 \mathrm{~Hz})$, 3.59 (s, $3 \mathrm{H}), 3.65(\mathrm{dd}, 1 \mathrm{H}, J=10.2,10.2 \mathrm{~Hz}), 4.16(\mathrm{ddd}, 1 \mathrm{H}, J=5.1,9.8$, $10.2 \mathrm{~Hz}), 4.27(\mathrm{dd}, 1 \mathrm{H}, J=5.1,10.2 \mathrm{~Hz}), 4.69(\mathrm{brd}, 1 \mathrm{H}, J=4.6 \mathrm{~Hz}), 5.49(\mathrm{~s}$, $1 \mathrm{H}), 6.05$ (brs, $1 \mathrm{H}, \mathrm{NH}), 7.33-7.39(\mathrm{~m}, 3 \mathrm{H}), 7.46-7.49$ (m, $2 \mathrm{H}) ;{ }^{13} \mathrm{C}$ NMR $\left(\mathrm{CDCl}_{3}, 100 \mathrm{MHz}\right): 23.1,36.9,55.3,56.2,58.8,62.5,69.6,84.1,98.5,102.1$, 126.2, 128.1, 128.9, 137.6; IR (ATR): 2968, 2933, 2860, 1456, 1375, 1132, 1082, $1043,760,698 \mathrm{~cm}^{-1}$. Anal. calcd for $\mathrm{C}_{16} \mathrm{H}_{23} \mathrm{NO}_{5}$ : C, 62.12; $\mathrm{H}, 7.49 ; \mathrm{N}, 4.53$. Found: C, 62.31; H, 7.69; N, 4.48

Methyl 4,6-O-benzylidene-2,3-dideoxy-3-( $N$ methoxytrifluoroacetamido)-3-C-methyl- $\alpha$-D-ribo-hexopyranoside (4)

To a solution of the crude amine $3(11.4 \mathrm{~g})$, pyridine $(5.9 \mathrm{ml}, 73 \mathrm{mmol})$ and DMAP $(0.20 \mathrm{~g}, 1.6 \mathrm{mmol})$ in $\mathrm{CH}_{2} \mathrm{Cl}_{2}(100 \mathrm{ml})$, trifluoroacetic anhydride $(10.2 \mathrm{ml}, 73.4 \mathrm{mmol})$ was added dropwise at $0^{\circ} \mathrm{C}$. The mixture was stirred at $0{ }^{\circ} \mathrm{C}$ for $0.5 \mathrm{~h}$, and quenched with water and then $1 \mathrm{M} \mathrm{HCl}$. The products were extracted with $\mathrm{CH}_{2} \mathrm{Cl}_{2}(3 \times)$. The combined organic extracts were successively washed with brine, saturated aqueous $\mathrm{NaHCO}_{3}$ and brine, and then dried $\left(\mathrm{Na}_{2} \mathrm{SO}_{4}\right)$, filtered and concentrated. The residue was triturated with (hexane/EtOAc $=9 / 1)$ to give trifluoroacetamide $4(10.9 \mathrm{~g})$ as white solids. The mother liquer was concentrated, and the residue was purified by column chromatography (hexane/EtOAc $=9 / 1$ ) to give $2.34 \mathrm{~g}$ of 4 . The combined yield of these materials was $95 \%$ in two steps. An analytical sample of 4 was obtained by recrystallization from $\mathrm{Et}_{2} \mathrm{O} /$ hexane as colorless prisms

$\mathrm{R}_{f} 0.52$ (hexane/EtOAc $=7 / 3$ ); m.p. $119-120^{\circ} \mathrm{C}\left(\mathrm{Et}_{2} \mathrm{O} /\right.$ hexane); $[\alpha]_{\mathrm{D}}^{28}+138$ (c 1.06, $\left.\mathrm{CHCl}_{3}\right) ;{ }^{1} \mathrm{H} \mathrm{NMR}\left(\mathrm{CDCl}_{3}, 400 \mathrm{MHz}\right): 1.53(\mathrm{~s}, 3 \mathrm{H}), 1.65(\mathrm{dd}, 1 \mathrm{H}$, $I=3.7,15.4 \mathrm{~Hz}), 3.27(\mathrm{~s}, 3 \mathrm{H}), 3.48(\mathrm{dd}, 1 \mathrm{H}, J=1.0,15.4 \mathrm{~Hz}), 3.61-3.69(\mathrm{~m}$, $2 \mathrm{H}), 3.81(\mathrm{~s}, 3 \mathrm{H}), 4.36(\mathrm{dd}, 1 \mathrm{H}, J=5.6,10.7 \mathrm{~Hz}), 4.54(\mathrm{ddd}, 1 \mathrm{H}, J=5.6,10.0$, $10.0 \mathrm{~Hz}), 4.63$ (brd, $1 \mathrm{H}, J=3.7 \mathrm{~Hz}), 5.53(\mathrm{~s}, 1 \mathrm{H}), 7.37-7.41(\mathrm{~m}, 3 \mathrm{H}), 7.49-7.52$ $(\mathrm{m}, 2 \mathrm{H}) ;{ }^{13} \mathrm{C} \mathrm{NMR}\left(\mathrm{CDCl}_{3}, 100 \mathrm{MHz}\right): 24.1,35.4,54.7,59.9,63.4,68.5,69.8$, $85.7,97.8,102.8,116.2\left(\mathrm{q}, J_{\mathrm{CF}}=289 \mathrm{~Hz}\right), 126.1,128.2,129.1,137.3,157.5(\mathrm{q}$, $J_{\mathrm{CF}}=37 \mathrm{~Hz}$ ); IR (ATR): 2950, 2837, 1701, 1379, 1203, 1153, 1115, 1066, 903, $746,700 \mathrm{~cm}^{-1}$. Anal. calcd for $\mathrm{C}_{18} \mathrm{H}_{22} \mathrm{~F}_{3} \mathrm{NO}_{6}$ : $\mathrm{C}, 53.33 ; \mathrm{H}, 5.47 ; \mathrm{N}, 3.46$. Found: C, 53.11; H, 5.77; N, 3.45 .

Methyl 4,6-O-benzylidene-2,3-dideoxy-3-C-methyl-3trifluoroacetamido- $\alpha$-D-ribo-hexopyranoside (5)

To a solution of trifluoroacetamide $4(6.99 \mathrm{~g}, 17.3 \mathrm{mmol})$ in $\mathrm{MeOH}(30 \mathrm{ml})$, $\mathrm{SmI}_{2}$ (prepared from $\mathrm{Sm}(10.2 \mathrm{~g}, 68.1 \mathrm{mmol})$ and 1,2-diiodoethane $(17 \mathrm{~g}$, $60.5 \mathrm{mmol})$ in THF $(600 \mathrm{ml}))^{35}$ was added at $0{ }^{\circ} \mathrm{C}$. After stirring for $0.5 \mathrm{~h}$, the reaction was quenched by the addition of $2 \mathrm{M} \mathrm{HCl}$, and the products extracted with EtOAc $(3 \times)$. The combined extracts were successively washed with saturated aqueous $\mathrm{NaHCO}_{3}, 10 \%$ aqueous $\mathrm{Na}_{2} \mathrm{~S}_{2} \mathrm{O}_{3}$ and brine, and then dried $\left(\mathrm{Na}_{2} \mathrm{SO}_{4}\right)$. After filtration and concentration in vacuo, the crude residue was purified by column chromatography (hexane/EtOAc $=75 / 25)$ to give amide $5(6.13 \mathrm{~g}$, $95 \%)$ as white solids. An analytical sample of $\mathbf{5}$ was obtained by crystallization from EtOAc/hexane as colorless prisms.

$\mathrm{R}_{f} 0.43$ (hexane/EtOAc $=7 / 3$ ); m.p. $120-121^{\circ} \mathrm{C}\left(\right.$ EtOAc/hexane); $[\alpha]_{\mathrm{D}}^{25}+73$ (c $\left.1.01, \mathrm{CHCl}_{3}\right) ;{ }^{1} \mathrm{H}$ NMR $\left(\mathrm{CDCl}_{3}, 400 \mathrm{MHz}\right): 1.58(\mathrm{~s}, 3 \mathrm{H}), 1.70(\mathrm{dd}, 1 \mathrm{H}$, $J=3.9,15.1 \mathrm{~Hz}$ ), $2.99(\mathrm{dd}, 1 \mathrm{H}, J=0.5,15.1 \mathrm{~Hz}), 3.33$ (s, 3H), 3.51 (d, $1 \mathrm{H}$, $J=9.8 \mathrm{~Hz}), 3.76(\mathrm{dd}, 1 \mathrm{H}, J=10.0,10.4 \mathrm{~Hz}), 3.95(\mathrm{ddd}, 1 \mathrm{H}, J=4.9,9.8$, $10.0 \mathrm{~Hz}), 4.30(\mathrm{dd}, 1 \mathrm{H}, J=4.9,10.4 \mathrm{~Hz}), 4.71(\mathrm{brd}, 1 \mathrm{H}, J=3.9 \mathrm{~Hz}), 5.61(\mathrm{~s}$, $1 \mathrm{H}), 7.14$ (brs, $1 \mathrm{H}, \mathrm{NH}), 7.34-7.41(\mathrm{~m}, 3 \mathrm{H}), 7.45-7.48(\mathrm{~m}, 2 \mathrm{H}) ;{ }^{13} \mathrm{C}$ NMR $\left(\mathrm{CDCl}_{3}, 100 \mathrm{MHz}\right): 22.9,37.2,53.5,55.1,59.6,69.0,82.9,98.1,101.9,115.6$ (q, $\left.J_{\mathrm{CF}}=291 \mathrm{~Hz}\right), 125.9,128.3,129.1,136.8,156.4\left(\mathrm{q}, J_{\mathrm{CF}}=36 \mathrm{~Hz}\right)$; IR (ATR): 3346, 3066, 2939, 2841, 1736, 1545, 1379, 1213, 1159, 1047, 903, 752, $698 \mathrm{~cm}^{-1}$. Anal. calcd for $\mathrm{C}_{17} \mathrm{H}_{20} \mathrm{~F}_{3} \mathrm{NO}_{5}$ : C, 54.40; $\mathrm{H}, 5.37 ; \mathrm{N}, 3.73$. Found: $\mathrm{C}, 54.16 ; \mathrm{H}$, 5.66; N, 3.77. Crystallographic data: $\mathrm{C}_{17} \mathrm{H}_{20} \mathrm{~F}_{3} \mathrm{NO}_{5}, \mathrm{MW}=375.34 \mathrm{Da}, 0.30 \times$ $0.30 \times 0.10 \mathrm{~mm}$, orthorhombic, space group $P=212121, Z=4, T=173(2) \mathrm{K}$, $a=9.6053(5), \quad b=12.3369(6), \quad c=15.1350(7) \AA, \quad V=1793.49(14) \AA^{3}, \quad \lambda($ Mo $\mathrm{K} \alpha)=0.71075 \AA, \mu=0.121 \mathrm{~mm}^{-1}$. Intensity data were collected on Rigaku R-AXIS Rapid IP area detector system (Rigaku, Tokyo, Japan). The structure was solved by direct methods and refined by the full-matrix least-squares on $F^{2}$ 
(SHELXL-97 (Sheldrick, 1997)). A total of 17480 reflections were measured and 4058 were found to be independent. Final $R 1=0.0386, w R 2=0.0967$ (3356 references; $I>2 \sigma(I)$ ), and goodness of fit $(\mathrm{GOF})=1.098$ (for all data, $R 1=0.0474, w R 2=0.1021)$.

\section{Methyl 4-O-benzoyl-6-bromo-2,3,6-trideoxy-3-C-methyl-3- trifluoroacetamido- $\alpha$-D-ribo-hexopyranoside (6)}

To a mixture of trifluoroacetamide $\mathbf{5}(10.0 \mathrm{~g}, 26.7 \mathrm{mmol})$ in dry carbon tetrachloride $(900 \mathrm{ml}), \mathrm{N}$-bromosuccinimide $(5.72 \mathrm{~g}, 32.1 \mathrm{mmol})$ and pyridine $(5 \mathrm{ml}, 61.8 \mathrm{mmol})$ were added. The mixture was heated under reflux for $4 \mathrm{~h}$ under normal room light illumination. Solvents were removed in vacuo to some extent, and the residue was dissolved in $\mathrm{CH}_{2} \mathrm{Cl}_{2}$ and washed successively with $5 \%$ aqueous $\mathrm{NaHSO}_{3}$, saturated aqueous $\mathrm{NaHCO}_{3}$ and brine, and then dried $\left(\mathrm{Na}_{2} \mathrm{SO}_{4}\right)$. After filtration and concentration in vacuo, the residue was purified by column chromatography (hexane/EtOAc $=9 / 1)$ to give bromide $\mathbf{6}(10.5 \mathrm{~g}, 87 \%)$ as white solids. Recrystallization from $\mathrm{Et}_{2} \mathrm{O} /$ hexane gave $\mathbf{6}$ as colorless prisms.

$\mathrm{R}_{f} 0.65$ (hexane/EtOAc $\left.=7 / 3\right) ;$ m.p. $118-119^{\circ} \mathrm{C}\left(\mathrm{Et}_{2} \mathrm{O} /\right.$ hexane $) ;[\alpha]_{\mathrm{D}}^{29}-14(c$ 1.02, $\left.\mathrm{CHCl}_{3}\right) ;{ }^{1} \mathrm{H}$ NMR $\left(\mathrm{CDCl}_{3}, 400 \mathrm{MHz}\right): 1.68$ (s, 3H), 1.94 (dd, $1 \mathrm{H}, J=3.8$, $15.1 \mathrm{~Hz}), 2.29(\mathrm{~d}, 1 \mathrm{H}, J=15.1 \mathrm{~Hz}), 3.41-3.51(\mathrm{~m}, 2 \mathrm{H}), 3.52(\mathrm{~s}, 3 \mathrm{H}), 4.10(\mathrm{ddd}$, $1 \mathrm{H}, J=2.7,7.7,10.2 \mathrm{~Hz}), 4.94(\mathrm{~d}, 1 \mathrm{H}, J=3.8 \mathrm{~Hz}), 5.12(\mathrm{~d}, 1 \mathrm{H}, J=10.2 \mathrm{~Hz}), 7.45$ $7.49(\mathrm{~m}, 2 \mathrm{H}), 7.58-7.62(\mathrm{~m}, 1 \mathrm{H}), 8.00-8.02(\mathrm{~m}, 2 \mathrm{H}), 8.24$ (brs, $1 \mathrm{H}, \mathrm{NH}) ;{ }^{13} \mathrm{C}$ NMR $\left(\mathrm{CDCl}_{3}, 100 \mathrm{MHz}\right): 23.3,32.1,40.8,55.5,56.4,67.1,74.8,97.7,115.8(\mathrm{q}$ $\left.J_{\mathrm{CF}}=291 \mathrm{~Hz}\right), 128.5,130.1,133.6,156.1\left(\mathrm{q}, J_{\mathrm{CF}}=36 \mathrm{~Hz}\right), 165.7$; IR (ATR): 3334, $3072,2943,2841,1739,1558,1450,1263,1217,1178,1045,960,877,710 \mathrm{~cm}^{-1}$. Anal. calcd for $\mathrm{C}_{17} \mathrm{H}_{19} \mathrm{BrF}_{3} \mathrm{NO}_{5}$ : C, 44.95; H, 4.22; N, 3.08. Found: C, 44.73; $\mathrm{H}$, 4.51; N, 3.01. Crystallographic data: $\mathrm{C}_{17} \mathrm{H}_{19} \mathrm{BrF}_{3} \mathrm{NO}_{5}, \mathrm{MW}=454.24 \mathrm{Da}, 0.60 \times$ $0.30 \times 0.20 \mathrm{~mm}$, monoclinic, space group $P=21, \quad Z=2, \quad T=168(2) \mathrm{K}$ $a=10.2351(6), b=7.6213(5), c=13.1952(7) \AA, V=967.64(10) \AA^{3}, \lambda($ Mo K $\alpha)=$ $0.71075 \AA, \mu=2.179 \mathrm{~mm}^{-1}$. Intensity data were collected on Rigaku R-AXIS Rapid IP area detector system. The structure was solved by direct methods and refined by the full-matrix least-squares on $F^{2}$ (SHELXL-97). A total of 9422 reflections were measured and 4368 were independent. Final $R 1=0.0340, w R 2=0.0774(3810$ references; $I>2 \sigma(I)$ ), and $\mathrm{GOF}=1.069$ (for all data, $R 1=0.0432, w R 2=0.0850$ ).

\section{Methyl 4-O-benzoyl-2,3,6-trideoxy-3-C-methyl-3- trifluoroacetamido- $\alpha$-D-erythro-hex-5-enopyranoside (7)}

To a solution of bromide $6(19.7 \mathrm{~g}, 43.3 \mathrm{mmol})$ in DMF $(220 \mathrm{ml})$, DBU $\left(12.2 \mathrm{ml}, 86.5 \mathrm{mmol}\right.$ ) was added. The mixture was heated to $90^{\circ} \mathrm{C}$ for $4 \mathrm{~h}$. After cooling to room temperature, the mixture was quenched with $10 \%$ aqueous $\mathrm{KHSO}_{4}$. The products were extracted with EtOAc $(3 \times)$, and the combined extracts were washed with saturated aqueous $\mathrm{NaHCO}_{3}$ and brine, and then dried $\left(\mathrm{Na}_{2} \mathrm{SO}_{4}\right)$. After filtration and concentration in vacuo, the residue was purified by column chromatography (hexane/EtOAc $=9 / 1$ ) to give enol ether 7 $(13 \mathrm{~g}, 80 \%)$ as white solids. Recrystallization from $\mathrm{Et}_{2} \mathrm{O} /$ hexane gave 7 as colorless prisms.

$\mathrm{R}_{f} 0.61$ (hexane/EtOAc $\left.=7 / 3\right) ;$ m.p. $117-118^{\circ} \mathrm{C}\left(\mathrm{Et}_{2} \mathrm{O} /\right.$ hexane $) ;[\alpha]_{\mathrm{D}}^{28}+29$ (c $\left.0.92, \mathrm{CHCl}_{3}\right) ;{ }^{1} \mathrm{H} \mathrm{NMR}\left(\mathrm{CDCl}_{3}, 400 \mathrm{MHz}\right): 1.69(\mathrm{~s}, 3 \mathrm{H}), 2.07(\mathrm{dd}, 1 \mathrm{H}$, $J=4.0,14.9 \mathrm{~Hz}), 2.53(\mathrm{dd}, 1 \mathrm{H}, J=1.0,14.9 \mathrm{~Hz}), 3.48(\mathrm{~s}, 3 \mathrm{H}), 4.69(\mathrm{dd}, 1 \mathrm{H}$, $J=1.7,1.7 \mathrm{~Hz}), 4.85(\mathrm{dd}, 1 \mathrm{H}, J=1.7,1.7 \mathrm{~Hz}), 4.96(\mathrm{brd}, 1 \mathrm{H}, J=4.0 \mathrm{~Hz}), 5.55$ (dd, $1 \mathrm{H}, J=1.7,1.7 \mathrm{~Hz}), 7.48-7.52(\mathrm{~m}, 2 \mathrm{H}), 7.60-7.64(\mathrm{~m}, 1 \mathrm{H}), 7.77(\mathrm{brs}, 1 \mathrm{H}$, $\mathrm{NH}), 8.09-8.11(\mathrm{~m}, 2 \mathrm{H}) ;{ }^{13} \mathrm{C} \mathrm{NMR}\left(\mathrm{CDCl}_{3}, 100 \mathrm{MHz}\right): 22.7,39.3,55.5,56.7$, $74.2,98.0,99.4,115.8\left(\mathrm{q}, J_{\mathrm{CF}}=291 \mathrm{~Hz}\right), 128.6,128.7,130.0,133.6,150.0,156.5$ $\left(\mathrm{q}, J_{\mathrm{CF}}=36 \mathrm{~Hz}\right), 165.3$; IR (ATR): 3332, 3072, 2941, 2843, 1736, 1662, 1560 , $1452,1267,1215,1161,1113,1045,987,870,712,677 \mathrm{~cm}^{-1}$. Anal. calcd for $\mathrm{C}_{17} \mathrm{H}_{18} \mathrm{~F}_{3} \mathrm{NO}_{5}$ : C, 54.69; H, 4.86; N, 3.75. Found: C, 54.93; H, 5.12; N, 3.90.

\footnotetext{
Methyl 4-O-benzoyl-2,3,6-trideoxy-3-C-methyl-3trifluoroacetamido- $\beta$-L-lyxo-hexopyranoside (8)

A mixture of enol ether $7(10.3 \mathrm{~g}, 27.6 \mathrm{mmol})$ and $\mathrm{RhCl}\left(\mathrm{PPh}_{3}\right)_{3}(1.20 \mathrm{~g}$, $1.30 \mathrm{mmol})$ in toluene $(250 \mathrm{ml})$ and $\mathrm{EtOH}(25 \mathrm{ml})$ was stirred under a hydrogen atmosphere (balloon) at room temperature for $10 \mathrm{~h}$. After filtration through a Cerite pad followed by concentration in vacuo, purification by column chromatography (hexane/EtOAc $=9 / 1 \rightarrow 7 / 3)$ gave benzoate $8(9.94 \mathrm{~g}$, $96 \%)$ as a colorless syrup and the stereoisomer epi-8 (0.31 g, 3\%) as white solids.
}

$\mathrm{R}_{f} 0.37$ (hexane/EtOAc $\left.=7 / 3\right) ;[\alpha]_{\mathrm{D}}^{29}+15$ (c 1.05, $\left.\mathrm{CHCl}_{3}\right) ;{ }^{1} \mathrm{H}$ NMR $\left(\mathrm{CDCl}_{3}, 400 \mathrm{MHz}\right): 1.29(\mathrm{~d}, 3 \mathrm{H}, J=6.3 \mathrm{~Hz}), 1.74(\mathrm{~s}, 3 \mathrm{H}), 1.93(\mathrm{dd}, 1 \mathrm{H}$ $J=9.5,15.1 \mathrm{~Hz}), 2.45(\mathrm{dd}, 1 \mathrm{H}, J=2.2,15.1 \mathrm{~Hz}), 3.56(\mathrm{~s}, 3 \mathrm{H}), 4.03(\mathrm{dq}, 1 \mathrm{H}$ $J=1.0,6.3 \mathrm{~Hz}$ ), 4.63 (dd, $1 \mathrm{H}, J=2.2,9.5 \mathrm{~Hz}), 5.14$ (brs, $1 \mathrm{H}), 6.79$ (brs, $1 \mathrm{H}$, $\mathrm{NH}), 7.45-7.49(\mathrm{~m}, 2 \mathrm{H}), 7.59-7.64(\mathrm{~m}, 1 \mathrm{H}), 8.11-8.13(\mathrm{~m}, 2 \mathrm{H}) ;{ }^{13} \mathrm{C} \mathrm{NMR}$ $\left(\mathrm{CDCl}_{3}, 100 \mathrm{MHz}\right): 17.3,21.4,37.1,56.7,57.1,68.2,73.4,99.4,115.2(\mathrm{q}$ $\left.J_{\mathrm{CF}}=291 \mathrm{~Hz}\right), 128.5,128.6,130.0,133.8,156.1\left(\mathrm{q}, J_{\mathrm{CF}}=37 \mathrm{~Hz}\right), 167.3$; IR (neat): $3336,3074,2989,2846,1728,1556,1452,1271,1203,1070,739$ $714 \mathrm{~cm}^{-1}$. Anal. calcd for $\mathrm{C}_{17} \mathrm{H}_{20} \mathrm{~F}_{3} \mathrm{NO}_{5}$ : $\mathrm{C}, 54.40 ; \mathrm{H}, 5.37 ; \mathrm{N}, 3.73$. Found: $\mathrm{C}$, $54.34 ; \mathrm{H}, 5.58 ; \mathrm{N}, 3.57$.

\section{Methyl 4-O-benzoyl-2,3,6-trideoxy-3-C-methyl-3-} trifluoroacetamido- $\alpha$-D-ribo-hexopyranoside (epi-8)

$\mathrm{R}_{f} 0.60$ (hexane/EtOAc $=7 / 3$ ); m.p. $104-105^{\circ} \mathrm{C}$ (colorless plates from $\left.\mathrm{MeOH}\right)$; $[\alpha]_{\mathrm{D}}^{20}-6.4\left(c \quad 0.89, \mathrm{CHCl}_{3}\right) ;{ }^{1} \mathrm{H}$ NMR $\left(\mathrm{CDCl}_{3}, 400 \mathrm{MHz}\right): 1.22(\mathrm{~d}, 3 \mathrm{H}, J=6.0$ $\mathrm{Hz}), 1.65$ (s, 3H), 1.89 (dd, $1 \mathrm{H}, J=4.1,15.1 \mathrm{~Hz}), 2.28$ (brd, $1 \mathrm{H}, J=15.1 \mathrm{~Hz}$ ), $3.44(\mathrm{~s}, 3 \mathrm{H}), 4.03(\mathrm{dq}, 1 \mathrm{H}, J=10.1,6.0 \mathrm{~Hz}), 4.83(\mathrm{brd}, 1 \mathrm{H}, J=4.1 \mathrm{~Hz}), 4.98(\mathrm{~d}$ $1 \mathrm{H}, J=10.1 \mathrm{~Hz}), 7.26-7.47(\mathrm{~m}, 2 \mathrm{H}), 7.56-7.59(\mathrm{~m}, 1 \mathrm{H}), 8.00-8.02(\mathrm{~m}, 2 \mathrm{H})$, 8.26 (brs, $1 \mathrm{H}, \mathrm{NH}) ;{ }^{13} \mathrm{C} \mathrm{NMR}\left(\mathrm{CDCl}_{3}, 100 \mathrm{MHz}\right): 17.5,23.3,40.9,55.2,56.3$, 63.1, 77.5, 97.7, $116.0\left(\mathrm{q}, J_{\mathrm{CF}}=295 \mathrm{~Hz}\right), 128.4,129.1,130.0,130.1,156.1$ $\left(\mathrm{q}, J_{\mathrm{CF}}=36 \mathrm{~Hz}\right), 166.1$; IR (ATR): 3348, 2937, 1737, 1715, 1583, 1556, 1450, $1323,1266,1216,1181,1128,1027,993,963,879,708 \mathrm{~cm}^{-1}$. Anal. calcd for $\mathrm{C}_{17} \mathrm{H}_{20} \mathrm{~F}_{3} \mathrm{NO}_{5}$ : C, 54.40; H, 5.37; N, 3.73. Found: C, 54.18; H, 5.13; $\mathrm{N}, 3.44$

\section{4-O-Benzoyl-2,3,6-trideoxy-3-C-methyl-3-trifluoroacetamido-} $\alpha, \beta$-L-lyxo-hexopyranosyl acetate (9)

Benzoate 8 ( $756 \mathrm{mg}, 2 \mathrm{mmol})$ was dissolved in $20 \%$ aqueous $\mathrm{AcOH}(40 \mathrm{ml})$, and the mixture was heated under reflux for $3.5 \mathrm{~h}$. After cooling to $0{ }^{\circ} \mathrm{C}$, the mixture was basified by saturated aqueous $\mathrm{NaHCO}_{3}$. After extraction (EtOAc, $3 \times$ ), the combined organic extracts were washed with brine and then dried $\left(\mathrm{Na}_{2} \mathrm{SO}_{4}\right)$. After filtration and evaporation in vacuo, the residue was dissolved in pyridine $(8 \mathrm{ml})$, to which was added $\mathrm{Ac}_{2} \mathrm{O}(2 \mathrm{ml})$, and DMAP $(6 \mathrm{mg})$ at $0{ }^{\circ} \mathrm{C}$. After $11 \mathrm{~h}$ at room temperature, the reaction was quenched by $1 \mathrm{M} \mathrm{HCl}$. After extraction $(\mathrm{EtOAc}, 3 \times$ ), the combined organic extracts were sequentially washed with brine, saturated aqueous $\mathrm{NaHCO}_{3}$ and brine, and then dried $\left(\mathrm{Na}_{2} \mathrm{SO}_{4}\right)$. After filtration and concentration in vacuo, purification with column chromatography (hexane/EtOAc $=6 / 4)$ gave acetate $9(715 \mathrm{mg}, 88 \%$, $\alpha / \beta=1 / 5)$ as white solids. Recrystallization from $\mathrm{CH}_{2} \mathrm{Cl}_{2} /$ hexane gave 9 as white powders.

$\mathrm{R}_{f} 0.45$ (hexane/EtOAc $\left.=6 / 4\right) ;$ m.p. $168-169^{\circ} \mathrm{C}\left(\mathrm{CH}_{2} \mathrm{Cl}_{2} /\right.$ hexane); ${ }^{1} \mathrm{H}$ NMR $\left(\mathrm{CDCl}_{3}, 400 \mathrm{MHz}\right)$ for the $\beta$-anomer: $1.29(\mathrm{~d}, 3 \mathrm{H}, J=6.3 \mathrm{~Hz}), 1.77(\mathrm{~s}, 3 \mathrm{H})$ $2.10(\mathrm{dd}, 1 \mathrm{H}, J=10.3,12.6 \mathrm{~Hz}), 2.15(\mathrm{~s}, 3 \mathrm{H}), 2.61(\mathrm{dd}, 1 \mathrm{H}, J=2.7,12.6 \mathrm{~Hz})$ $4.17(\mathrm{dq}, 1 \mathrm{H}, J=1.0,6.3 \mathrm{~Hz}$ ), 5.03 (brs, $1 \mathrm{H}), 5.96$ (dd, $1 \mathrm{H}, J=2.7,10.3 \mathrm{~Hz}$ ) 6.99 (brs, $1 \mathrm{H}, \mathrm{NH}), 7.48-7.67(\mathrm{~m}, 3 \mathrm{H}), 8.13-8.16(\mathrm{~m}, 2 \mathrm{H})$; for the selected $\alpha$-anomer: $1.25(\mathrm{~d}, 3 \mathrm{H}, J=6.9 \mathrm{~Hz}), 4.38(\mathrm{q}, 1 \mathrm{H}, J=6.9 \mathrm{~Hz}), 5.07$ (brs, $1 \mathrm{H})$ 6.34 (brd, $1 \mathrm{H}, J=4.0 \mathrm{~Hz}), 7.10$ (brs, $1 \mathrm{H}, \mathrm{NH}) ;{ }^{13} \mathrm{C} \mathrm{NMR}\left(\mathrm{CDCl}_{3}, 100 \mathrm{MHz}\right)$ $17.2,21.0,21.2,35.3,57.2,69.1,73.4,90.6,115.2\left(\mathrm{q}, J_{\mathrm{CF}}=290 \mathrm{~Hz}\right), 128.3$ 128.7, 130.0, 134.1, 156.3 (q, $J_{\mathrm{CF}}=37 \mathrm{~Hz}$ ), 167.7, 169.1; IR (ATR): 3334, 3076 $2989,1728,1556,1452,1273,1161,1049,908,758,715 \mathrm{~cm}^{-1}$. Anal. calcd for $\mathrm{C}_{18} \mathrm{H}_{20} \mathrm{~F}_{3} \mathrm{NO}_{6}$ : C, 53.60; H, 5.00; N, 3.47. Found: C, 53.39; H, 5.25; N, 3.37.

\section{Methyl 2,3,6-trideoxy-3-C-methyl-3-trifluoroacetamido- $\beta$-L-lyxo- hexopyranoside (10)}

To a solution of $\mathrm{Mg}(\mathrm{OMe})_{2}$ in $\mathrm{MeOH}$ (prepared from magnesium $(1.12 \mathrm{~g}$, $46 \mathrm{mmol})$ and $\mathrm{MeOH}(230 \mathrm{ml})$ ), a solution of benzoate $8(6.24 \mathrm{~g}, 16.6 \mathrm{mmol})$ in $\mathrm{MeOH}(100 \mathrm{ml})$ was added at $0{ }^{\circ} \mathrm{C}$. After stirring for $1.5 \mathrm{~h}$, the mixture was acidified by carefully adding aqueous $2 \mathrm{M} \mathrm{HCl}$, and $\mathrm{MeOH}$ was removed under reduced pressure. The products were extracted with EtOAc $(5 \times)$, and the combined organic extracts were washed with brine and then dried $\left(\mathrm{Na}_{2} \mathrm{SO}_{4}\right)$. After filtration, solvents were evaporated in vacuo, and purification by column chromatography (hexane/EtOAc = 8/2) gave alcohol $10(3.75 \mathrm{~g}, 83 \%)$ as white solids. Recrystallization from $\mathrm{Et}_{2} \mathrm{O}$ gave $\mathbf{1 0}$ as colorless plates.

$\mathrm{R}_{f} 0.35$ (hexane/EtOAc $=7 / 3$ ); m.p. $104-106^{\circ} \mathrm{C}\left(\mathrm{Et}_{2} \mathrm{O}\right) ;[\alpha]_{\mathrm{D}}^{24}+56(c$ 1.1, $\left.\mathrm{CHCl}_{3}\right) ;{ }^{1} \mathrm{H}$ NMR $\left(\mathrm{CDCl}_{3}, 400 \mathrm{MHz}\right): 1.33(\mathrm{~d}, 3 \mathrm{H}, J=6.4 \mathrm{~Hz}), 1.61(\mathrm{~s}, 3 \mathrm{H})$, $1.62(\mathrm{dd}, 1 \mathrm{H}, J=9.2,14.0 \mathrm{~Hz}), 2.30(\mathrm{~d}, 1 \mathrm{H}, J=10.4 \mathrm{~Hz}), 2.46(\mathrm{dd}, 1 \mathrm{H}, J=2.0$, 
$14.0 \mathrm{~Hz}), 3.24(\mathrm{~d}, 1 \mathrm{H}, J=10.4 \mathrm{~Hz}), 3.49(\mathrm{~s}, 3 \mathrm{H}), 3.88(\mathrm{q}, 1 \mathrm{H}, J=6.4 \mathrm{~Hz}), 4.43$ (dd, $1 \mathrm{H}, J=2.0,9.2 \mathrm{~Hz}), 7.06(\mathrm{~s}, 1 \mathrm{H}, \mathrm{NH}) ;{ }^{13} \mathrm{C} \mathrm{NMR}\left(\mathrm{CDCl}_{3}, 100 \mathrm{MHz}\right): 17.0$, $20.3,36.4,56.6,56.7,68.5,72.3,100.0,115.5\left(\mathrm{q}, J_{\mathrm{CF}}=287 \mathrm{~Hz}\right), 156.2(\mathrm{q}$, $J_{\mathrm{CF}}=35 \mathrm{~Hz}$ ); IR (ATR): 3380, 2986, 2950, 1719, 1549, 1463, 1448, 1394, 1332, $1259,1187,1164,1145,1122,1104,1070,1006 \mathrm{~cm}^{-1}$. Anal. calcd for $\mathrm{C}_{10} \mathrm{H}_{16}$ $\mathrm{F}_{3} \mathrm{NO}_{4}$ : C, 44.28; H, 5.95; N, 5.16. Found: C, 44.50; H, 6.02; N, 4.86.

\section{Methyl 4-O-benzyl-2,3,6-trideoxy-3-C-methyl-3- trifluoroacetamido- $\beta$-L-lyxo-hexopyranoside (11)}

To a mixture of $\mathrm{NaH}$ (63\% dispersion in oil, $1.8 \mathrm{~g}, 48 \mathrm{mmol}$ ) in DMF $(50 \mathrm{ml})$, a solution of alcohol $10(3.75 \mathrm{~g}, 13.8 \mathrm{mmol})$ in DMF $(20 \mathrm{ml})$ was added. After stirring for $2 \mathrm{~h}$, benzyl bromide $(5.66 \mathrm{~g}, 33.1 \mathrm{mmol})$ was added, and the mixture was allowed to warm to room temperature. After stirring for $2 \mathrm{~h}$, water and $\mathrm{Et}_{2} \mathrm{NH}$ were successively added at $0{ }^{\circ} \mathrm{C}$. After $0.5 \mathrm{~h}$, aqueous $2 \mathrm{M} \mathrm{HCl}$ was added, and the products were extracted with $\mathrm{Et}_{2} \mathrm{O}(3 \times)$. Combined organic extracts were washed successively with brine, saturated aqueous $\mathrm{NaHCO}_{3}$ and brine, and then dried $\left(\mathrm{Na}_{2} \mathrm{SO}_{4}\right)$. After filtration and concentration in vacuo, the residue was purified by column chromatography (hexane/EtOAc $=8 / 2$ ) to give benzyl ether $11(4.52 \mathrm{~g}, 91 \%)$ as a colorless oil.

$\mathrm{R}_{f} 0.45$ (hexane/EtOAc $\left.=7 / 3\right) ;[\alpha]_{\mathrm{D}}^{29}-30\left(c 1.0, \mathrm{CHCl}_{3}\right) ;{ }^{1} \mathrm{H}$ NMR $\left(\mathrm{CDCl}_{3}\right.$, $400 \mathrm{MHz}): 1.40(\mathrm{~d}, 3 \mathrm{H}, J=6.4 \mathrm{~Hz}), 1.64(\mathrm{~s}, 3 \mathrm{H}), 1.80(\mathrm{dd}, 1 \mathrm{H}, J=9.2$, $12.4 \mathrm{~Hz}), 2.05(\mathrm{dd}, 1 \mathrm{H}, J=2.4,12.4 \mathrm{~Hz}), 3.44(\mathrm{~s}, 1 \mathrm{H}), 3.49(\mathrm{~s}, 3 \mathrm{H}), 3.86(\mathrm{q}, 1 \mathrm{H}$, $J=6.4 \mathrm{~Hz}), 4.48(\mathrm{dd}, 1 \mathrm{H}, J=2.4,9.2 \mathrm{~Hz}), 4.50(\mathrm{~d}, 1 \mathrm{H}, J=11.2 \mathrm{~Hz}), 4.81(\mathrm{~d}$, $1 \mathrm{H}, J=11.2 \mathrm{~Hz}), 6.42$ (brs, $1 \mathrm{H}, \mathrm{NH}), 7.29-7.38(\mathrm{~m}, 5 \mathrm{H}) ;{ }^{13} \mathrm{C} \mathrm{NMR}\left(\mathrm{CDCl}_{3}\right.$, $100 \mathrm{MHz}): 17.6,21.3,37.5,56.2,56.8,69.2,75.9,79.2,99.0,115.2\left(\mathrm{q}, J_{\mathrm{CF}}=287\right.$ $\mathrm{Hz}), 127.7,127.9,128.4,137.0,156.1\left(\mathrm{q}, J_{\mathrm{CF}}=36 \mathrm{~Hz}\right) \mathrm{cm}^{-1}$; IR (ATR): 3326 , 3091, 3068, 2989, 2938, 2877, 1723, 1553, 1520, 1498, 1454, 1397, 1371, 1355 , $1278,1258,1171,1128,1111,1071,1028,1016 \mathrm{~cm}^{-1}$. Anal. calcd for $\mathrm{C}_{17} \mathrm{H}_{22}$ $\mathrm{F}_{3} \mathrm{NO}_{4}$ : C, 57.55; H, 5.27; N, 2.82. Found: C, 57.71; H, 5.31; N, 2.58 .

\section{4-O-Benzyl-2,3,6-trideoxy-3-C-methyl-3-trifluoroacetamido-} $\alpha, \beta$-L-lyxo-hexopyranosyl acetate (12)

Benzyl ether $11(1.50 \mathrm{~g}, 4.15 \mathrm{mmol})$ was dissolved in $20 \%$ aqueous $\mathrm{AcOH}$ $(200 \mathrm{ml}, 0.68 \mathrm{~mol})$, and the mixture was heated under reflux for $3.5 \mathrm{~h}$. After cooling to $0{ }^{\circ} \mathrm{C}$, the mixture was basified by adding $\mathrm{KOH}(3 \mathrm{M}, 220 \mathrm{ml}$, $0.66 \mathrm{~mol}$ ) and saturated aqueous $\mathrm{NaHCO}_{3}$. After extraction with EtOAc $(3 \times)$, the combined organic extracts were washed with brine and then dried $\left(\mathrm{Na}_{2} \mathrm{SO}_{4}\right)$. After filtration, solvents were removed in vacuo. The residue was dissolved in pyridine $(5 \mathrm{ml})$, to which was added $\mathrm{Ac}_{2} \mathrm{O}(1 \mathrm{ml})$ and DMAP $\left(10 \mathrm{mg}\right.$ ) at $0{ }^{\circ} \mathrm{C}$. After $1.5 \mathrm{~h}$, the reaction was quenched by adding $1 \mathrm{M} \mathrm{HCl}$. The products were extracted with EtOAc $(3 \times)$, the combined organic extracts were washed successively with brine, saturated aqueous $\mathrm{NaHCO}_{3}$ and brine, and then dried $\left(\mathrm{Na}_{2} \mathrm{SO}_{4}\right)$. After filtration and concentration in vacuo, purification with column chromatography (hexane/EtOAc $=8 / 2)$ gave acetate $12(1.48 \mathrm{~g}$, $92 \% ; \alpha / \beta=45 / 55)$ as a colorless gummy syrup.

$\mathrm{R}_{f} 0.32$ (hexane/EtOAc $\left.=7 / 3\right) ;{ }^{1} \mathrm{H}$ NMR $\left(\mathrm{CDCl}_{3}, 400 \mathrm{MHz}\right)$ for the $\beta$-anomer: $1.41(\mathrm{~d}, 3 \mathrm{H}, J=6.6 \mathrm{~Hz}), 1.66(\mathrm{~s}, 3 \mathrm{H}), 2.04(\mathrm{dd}, 1 \mathrm{H}, J=9.0$, $12.4 \mathrm{~Hz}$ ), 2.08 (s, 3H), $2.11(\mathrm{dd}, 1 \mathrm{H}, J=2.9,12.4 \mathrm{~Hz}), 3.45(\mathrm{~s}, 1 \mathrm{H}), 4.02(\mathrm{q}, 1 \mathrm{H}$, $J=6.6 \mathrm{~Hz}), 4.51(\mathrm{~d}, 1 \mathrm{H} J=11.7 \mathrm{~Hz}), 4.83(\mathrm{~d}, 1 \mathrm{H}, J=11.7 \mathrm{~Hz}), 5.84(\mathrm{dd}, 1 \mathrm{H}$ $J=2.9,9.0 \mathrm{~Hz}), 6.51(\mathrm{~s}, 1 \mathrm{H}, \mathrm{NH}), 7.33-7.39(\mathrm{~m}, 5 \mathrm{H})$; for the selected $\alpha$ anomer: $1.39(\mathrm{~d}, 3 \mathrm{H}, J=6.6 \mathrm{~Hz}), 6.17(\mathrm{dd}, 1 \mathrm{H}, J=1.6,3.6 \mathrm{~Hz}) ;{ }^{13} \mathrm{C} \mathrm{NMR}$ $\left(\mathrm{CDCl}_{3}, 100 \mathrm{MHz}\right)$ for the $\alpha$ - and $\beta$-mixture: $17.6(2 \times), 21.0,21.2,21.4,22.8$, $34.1,35.9,54.9,56.4,66.5,70.2,76.0,76.1,79.0,79.8,90.5,90.9,115.3(\mathrm{q}$ $\left.J_{\mathrm{CF}}=291 \mathrm{~Hz}\right), 128.0,128.1,128.4(2 \times), 128.7,128.8,136.8,156.4\left(\mathrm{q}, J_{\mathrm{CF}}=37\right.$ $\mathrm{Hz}), 169.2$ (2×); IR (neat): 3338, 3092, 3068, 3033, 2988, 2939, 2920, 2883, $1754,1723,1556,1521,1498,1455,1386,1370,1355,1312,1232,1201,1187$, $1162,1130,1107,1085,1049,1007 \mathrm{~cm}^{-1}$. Anal. calcd for $\mathrm{C}_{18} \mathrm{H}_{22} \mathrm{~F}_{3} \mathrm{NO}_{5}$ : C, 55.53; H, 5.70; N, 3.60. Found: C, 55.83; H, 5.47; N, 3.42 .

\section{Methyl 4-O-benzyl-2,3,6-trideoxy-3- $C$-methyl-3-amino- $\beta$-L- lyxo-hexopyranoside (13)}

To a solution of amide $11(4.69 \mathrm{~g}, 13 \mathrm{mmol})$ in $\mathrm{MeOH}(55 \mathrm{ml}), 5 \mathrm{M} \mathrm{NaOH}$ $(14 \mathrm{ml}, 60 \mathrm{mmol})$ was added and the reaction mixture was stirred at room temperature for $9 \mathrm{~h}$. The reaction mixture was concentrated in vacuo to some extent, was diluted with $\mathrm{H}_{2} \mathrm{O}$ and EtOAc, and the was extracted with EtOAc $(5 \times)$. The combined organic extracts were washed with brine and then dried
$\left(\mathrm{Na}_{2} \mathrm{SO}_{4}\right)$, and concentration in vacuo gave amine $13(3.22 \mathrm{~g}, 93 \%)$ as a colorless oil.

$\mathrm{R}_{f} 0.24\left(\mathrm{CHCl}_{3} / \mathrm{MeOH}=9 / 1\right) ;[\alpha]_{\mathrm{D}}^{20}+2.5\left(c\right.$ 1.66, $\left.\mathrm{CHCl}_{3}\right) ;{ }^{1} \mathrm{H}$ NMR $\left(\mathrm{CDCl}_{3}, 500 \mathrm{MHz}\right): 1.17(\mathrm{~s}, 3 \mathrm{H}), 1.32(\mathrm{~d}, 3 \mathrm{H}, J=6.6 \mathrm{~Hz}), 1.55$ (dd, $1 \mathrm{H}, J=2.5$, $12.8 \mathrm{~Hz}$ ), $1.68(\mathrm{dd}, 1 \mathrm{H}, J=9.7,12.8 \mathrm{~Hz}), 2.08-2.25$ (brs, $2 \mathrm{H}, \mathrm{NH}), 2.89(\mathrm{~s}, 1 \mathrm{H})$, $3.47(\mathrm{~s}, 3 \mathrm{H}), 3.76(\mathrm{q}, 1 \mathrm{H}, J=6.6 \mathrm{~Hz}), 4.39(\mathrm{dd}, 1 \mathrm{H}, J=2.5,9.7 \mathrm{~Hz}), 4.68(\mathrm{~d}$, $1 \mathrm{H}, J=15.2 \mathrm{~Hz}), 4.77(\mathrm{~d}, 1 \mathrm{H}, J=15.2 \mathrm{~Hz}), 7.25-7.40(\mathrm{~m}, 5 \mathrm{H}) ;{ }^{13} \mathrm{C} \mathrm{NMR}$ $\left(\mathrm{CDCl}_{3}, 125 \mathrm{MHz}\right): 17.5,24.9,42.2,52.4,56.3,69.8,83.9,100.5,127.6,127.8$, $128.2,128.3,138.2 \mathrm{~cm}^{-1}$; IR (neat): $3361,2929,1722,1586,1497,1455,1392$, $1224,1164,1070,1011,965,876,755,704 \mathrm{~cm}^{-1}$. Anal. calcd for $\mathrm{C}_{15} \mathrm{H}_{23} \mathrm{NO}_{3}: \mathrm{C}$, 67.90; H, 8.74; N, 5.28. Found: C, 67.83; H, 9.03; N, 5.01.

Preparation of $\mathrm{TfN}_{3}$ in toluene $e^{44}$. To a mixture of $\mathrm{NaN}_{3}(5.92 \mathrm{~g}, 91.1 \mathrm{mmol})$ in toluene $(15 \mathrm{ml})$ and $\mathrm{H}_{2} \mathrm{O}(15 \mathrm{ml}), \mathrm{Tf}_{2} \mathrm{O}(7.76 \mathrm{ml}, 45.5 \mathrm{mmol})$ was added at $0{ }^{\circ} \mathrm{C}$; the mixture was stirred at $10{ }^{\circ} \mathrm{C}$ for $2 \mathrm{~h}$. The reaction mixture was quenched by the addition of saturated aqueous $\mathrm{NaHCO}_{3}$, and the mixture was extracted by toluene $(10 \mathrm{ml} 2 \times)$ to give a toluene solution of $\mathrm{TfN}_{3}$, which was used in the subsequent diazo-transfer reaction. (warning: $\mathrm{TfN}_{3}$ has an explosive nature and requires very careful treatment.)

\section{Methyl 4-O-benzyl-2,3,6-trideoxy-3-C-methyl-3-azido- $\beta$-L-lyxo- hexopyranoside (14)}

To a suspension of a mixture of amine $13(3.22 \mathrm{~g}, 12.2 \mathrm{mmol}), \mathrm{CuSO}_{4}(208 \mathrm{mg}$, $1.30 \mathrm{mmol}), \mathrm{NaHCO}_{3}(5.44 \mathrm{~g}, 64.8 \mathrm{mmol})$ in $\mathrm{MeOH}(28 \mathrm{ml})$ and $\mathrm{H}_{2} \mathrm{O}(7 \mathrm{ml})$, a freshly prepared $\mathrm{TfN}_{3}$ solution $(28 \mathrm{ml})$ was added at $0{ }^{\circ} \mathrm{C}$. After stirring for $2.5 \mathrm{~h}$ at room temperature, the mixture was diluted with $\mathrm{H}_{2} \mathrm{O}$ and EtOAc, and the products extracted with EtOAc $(3 \times)$. The combined organic extracts were washed with brine, and it was then dried $\left(\mathrm{Na}_{2} \mathrm{SO}_{4}\right)$ and concentrated in vacuo. The residue was purified by flash column chromatography (hexane/ $\mathrm{EtOAc}=85 / 15)$ to give azide $14(3.51 \mathrm{~g}, 99 \%)$ as white solids. Recrystallization from $\mathrm{MeOH}$ gave $\mathbf{1 4}$ as colorless needles.

$\mathrm{R}_{f} 0.45$ (hexane/EtOAc $\left.=4 / 1\right) ;$ m.p. $108-110^{\circ} \mathrm{C}(\mathrm{MeOH}) ;[\alpha]_{\mathrm{D}}^{28}+54.9$ (c 1.07, $\left.\mathrm{CHCl}_{3}\right)$; ${ }^{1} \mathrm{H} \mathrm{NMR}\left(\mathrm{CDCl}_{3}, 500 \mathrm{MHz}\right): 1.19$ (d, $\left.3 \mathrm{H}, J=6.3 \mathrm{~Hz}\right), 1.30$ $(\mathrm{s}, 3 \mathrm{H}), 1.77(\mathrm{dd}, 1 \mathrm{H}, J=2.3,12.6 \mathrm{~Hz}), 2.11(\mathrm{dd}, 1 \mathrm{H}, J=9.2,12.6 \mathrm{~Hz}), 3.01$ $(\mathrm{s}, 1 \mathrm{H}), 3.48(\mathrm{~s}, 3 \mathrm{H}), 3.67(\mathrm{q}, 1 \mathrm{H}, J=6.3 \mathrm{~Hz}), 4.45(\mathrm{dd}, 1 \mathrm{H}, J=2.3,9.2 \mathrm{~Hz})$, $4.57(\mathrm{~d}, 1 \mathrm{H}, J=11.5 \mathrm{~Hz}), 4.92(\mathrm{~d}, 1 \mathrm{H}, J=11.5 \mathrm{~Hz}), 7.28-7.37(\mathrm{~m}, 3 \mathrm{H})$, 7.39-7.43 (m, 2H); ${ }^{13} \mathrm{C}$ NMR $\left(\mathrm{CDCl}_{3}, 125 \mathrm{MHz}\right): 17.1,23.3,36.1,56.4,62.8$, 69.8, 75.8, 81.1, 99.8, 128.0, 128.3, 128.7, 137.5; IR (ATR): 2866, 2097, 1458, 1390, 1352, 1252, 1156, 1116, 1062, 1010, 977, 876, 813, 764, $697 \mathrm{~cm}^{-1}$. Anal. calcd for $\mathrm{C}_{15} \mathrm{H}_{21} \mathrm{~N}_{3} \mathrm{O}_{3}$ : C, 61.84; $\mathrm{H}, 7.27 ; \mathrm{N}, 14.42$. Found: C, 61.98; H, 7.11; $\mathrm{N}$, 14.31. Crystallographic data: $\mathrm{C}_{15} \mathrm{H}_{21} \mathrm{~N}_{3} \mathrm{O}_{3}, \quad \mathrm{MW}=291.35 \mathrm{Da}, 0.20 \times$ $0.15 \times 0.15 \mathrm{~mm}$, orthorhombic, space group $P=212121, Z=4, T=173(2)$ $\mathrm{K}, a=10.5718(13), b=11.5146(11), c=12.8642(14) \AA, V=1566(3) \AA^{3}, \lambda(\mathrm{Cu}$ $\mathrm{K} \alpha)=1.54187 \AA, \mu=0.713 \mathrm{~mm}^{-1}$. Intensity data were collected on Rigaku VariMax Rapid-II IP area detector system. The structure was solved by direct methods and refined by the full-matrix least-squares on $F^{2}$ (SHELXL-97). A total of 17995 reflections were measured, and 2852 were found to be independent. Final $R 1=0.0452, w R 2=0.1124$ (2399 references; $I>2 \sigma(I)$ ), and $\mathrm{GOF}=1.018$ (for all data, $R 1=0.0513, w R 2=0.1168$ ).

Alternative synthesis of 14 by using reagent 16. To a solution of amine 13 $(34.3 \mathrm{mg}, 0.13 \mathrm{mmol})$ and DMAP $(47.1 \mathrm{mg}, 0.39 \mathrm{mmol})$ in $\mathrm{MeCN}(1.3 \mathrm{ml}), 16$ $(45.2 \mathrm{mg}, 0.16 \mathrm{mmol})$ was added at $0{ }^{\circ} \mathrm{C}$. After stirring for $1 \mathrm{~h}$ at room temperature, the reaction was quenched with saturated aqueous $\mathrm{NaHCO}_{3}$ at $0{ }^{\circ} \mathrm{C}$, and the products were extracted with EtOAc $(3 \times)$. The combined organic extracts were successively washed with $\mathrm{H}_{2} \mathrm{O}$ and brine, and then dried $\left(\mathrm{Na}_{2} \mathrm{SO}_{4}\right)$. After filtration and concentration in vacuo, the crude residue was purified by preparative TLC (hexane/EtOAc $=8 / 2)$ to give azide $14(34.3 \mathrm{mg}$, 91\%) as white solids.

\section{4-O-Benzyl-2,3,6-trideoxy-3-C-methyl-3-azido- $\alpha, \beta$-L-lyxo- hexopyranosyl acetate (15)}

Azide $14(4.06 \mathrm{~g}, 13.9 \mathrm{mmol})$ was dissolved in $20 \%$ aqueous $\mathrm{AcOH}(250 \mathrm{ml})$, and the mixture was heated under reflux for $3.5 \mathrm{~h}$. After cooling to $0{ }^{\circ} \mathrm{C}$, the mixture was basified by adding $\mathrm{KOH}(46 \mathrm{~g})$ and saturated aqueous $\mathrm{NaHCO}_{3}$. After extraction (AcOEt, $3 \times$ ), the combined organic extracts were washed 
with brine and then dried $\left(\mathrm{Na}_{2} \mathrm{SO}_{4}\right)$. After filtration and evaporation in vacuo, the residue was dissolved in pyridine $(65 \mathrm{ml})$, to which was added $\mathrm{Ac}_{2} \mathrm{O}$ $(17 \mathrm{ml})$, and 4 -DMAP $(18 \mathrm{mg})$ at $0{ }^{\circ} \mathrm{C}$. After $11 \mathrm{~h}$ at room temperature, the reaction was quenched by $1 \mathrm{M} \mathrm{HCl}$. After extraction (EtOAc, $3 \times$ ), the combined organic extracts were sequentially washed with brine, saturated aqueous $\mathrm{NaHCO}_{3}$ and brine, and then dried $\left(\mathrm{Na}_{2} \mathrm{SO}_{4}\right)$. After filtration and concentration in vacuo, purification with column chromatography (hexane/ EtOAc $=8 / 2)$ gave azide acetate $15(3.63 \mathrm{~g}, 81 \% ; \alpha / \beta=1 / 2.5)$ as a colorless gummy syrup.

$\mathrm{R}_{f} 0.39$ (hexane/EtOAc $\left.=4 / 1\right)$ for the $\beta$-anomer, $\mathrm{R}_{f} 0.44$ (hexane/EtOAc $=$ $4 / 1)$ for the $\alpha$-anomer; ${ }^{1} \mathrm{H}$ NMR $\left(\mathrm{CDCl}_{3}, 500 \mathrm{MHz}\right)$ for the $\beta$-anomer: 1.19 (d, $3 \mathrm{H}, J=6.4 \mathrm{~Hz}), 1.35$ (s, 3H), 1.79 (dd, $1 \mathrm{H}, J=2.3,12.4 \mathrm{~Hz}), 2.10$ (s, $3 \mathrm{H})$, $2.29(\mathrm{dd}, 1 \mathrm{H}, J=9.6,12.4 \mathrm{~Hz}), 3.04(\mathrm{~s}, 1 \mathrm{H}), 3.82(\mathrm{dq}, 1 \mathrm{H}, J=1.4,6.4 \mathrm{~Hz})$ $4.59(\mathrm{~d}, 1 \mathrm{H} J=11.0 \mathrm{~Hz}), 4.92(\mathrm{~d}, 1 \mathrm{H}, J=11.0 \mathrm{~Hz}), 5.80(\mathrm{dd}, 1 \mathrm{H} J=2.3$, 9.6 Hz), 7.28-7.43 (m, 5H); for the selected $\alpha$-anomer: 1.79 (dd, $1 \mathrm{H}, J=1.4$, $13.7 \mathrm{~Hz}), 2.42(\mathrm{dd}, 1 \mathrm{H}, J=4,2,13.7 \mathrm{~Hz}), 3.17(\mathrm{~s}, 1 \mathrm{H}), 4.10(\mathrm{dq}, 1 \mathrm{H}, J=1.4$ $6.4 \mathrm{~Hz}), 6.24(\mathrm{dd}, 1 \mathrm{H}, J=1.4,4.2 \mathrm{~Hz}) ;{ }^{13} \mathrm{C} \mathrm{NMR}\left(\mathrm{CDCl}_{3}, 125 \mathrm{MHz}\right)$ for the $\alpha-$ and $\beta$-mixture: $17.1(2 \times), 21.1,21.2,23.0,24.2,33.4,34.8,61.0,62.4,67.5$, $70.8,75.6,75.7,80.6,81.0,91.2,91.5,127.9,128.3(2 \times), 128.4,128.6,137.5$, 137.6, 169.1, 169.3; IR (neat): 2982, 2938, 2099, 1750, 1455, 1368, 1235, 1208, $1168,1143,1103,1048,1003,917,756,700,598,531 \mathrm{~cm}^{-1}$. Anal. calcd for $\mathrm{C}_{16} \mathrm{H}_{21} \mathrm{~N}_{3} \mathrm{O}_{4}$ : C, 60.17; H, 6.63; N, 13.16. Found: C, 60.20; H, 6.36; N, 12.96 .

Typical procedure for C-glycosylation reaction. To a mixture of $\mathrm{Sc}(\mathrm{OTf})_{3}$ $(612 \mathrm{mg}, 1.24 \mathrm{mmol})$, phenol $17(2.02 \mathrm{~g}, 9.70 \mathrm{mmol})$ and powdered Drierite $(8.16 \mathrm{~g})$ in 1,2-dichloroethane $(40 \mathrm{ml})$, acetate $12(2.23 \mathrm{~g}, 5.73 \mathrm{~mol})$ in 1,2 dichloroethane $(10 \mathrm{ml})$ was added at $-30^{\circ} \mathrm{C}$. After gradual warming to $0{ }^{\circ} \mathrm{C}$, the mixture was quenched with saturated aqueous $\mathrm{NaHCO}_{3}$ at $0^{\circ} \mathrm{C}$. After filtration through a Celite pad, the products were extracted with $\mathrm{CH}_{2} \mathrm{Cl}_{2}$ $(3 \times)$. The combined organic extracts were washed with brine and then dried $\left(\mathrm{Na}_{2} \mathrm{SO}_{4}\right)$. After filtration, solvents were removed in vacuo, and the residue was purified by column chromatography (hexane/EtOAc $=95 / 5 \rightarrow 8 / 2$ ) to give C-glycoside $19(2.72 \mathrm{~g}, 88 \%)$ as a colorless oil.

\section{Methyl 2-hydroxy-3-(4-O-benzoyl-2,3,6-trideoxy-3-C-methyl-3- trifluoroacetamido- $\beta$-L-lyxo-hexopyranosyl)-6-(prop-2-ene-1- yloxy)benzoate (18)}

Colorless plates, 94\%; $\mathrm{R}_{f} 0.40$ (hexane/EtOAc $=3 / 1$ ); m.p. $71-73^{\circ} \mathrm{C}(\mathrm{EtOH} /$ hexane); $[\alpha]^{22}-65$ (c 1.0, $\left.\mathrm{CHCl}_{3}\right) ;{ }^{1} \mathrm{H}$ NMR $\left(\mathrm{CDCl}_{3}, 400 \mathrm{MHz}\right): 1.29$ (d, $3 \mathrm{H}$, $J=6.0 \mathrm{~Hz}$ ), $1.88(\mathrm{~s}, 3 \mathrm{H}), 1.95(\mathrm{dd}, 1 \mathrm{H}, J=11.6,12.4 \mathrm{~Hz}), 2.58(\mathrm{dd}, 1 \mathrm{H}, J=2.0$, $12.4 \mathrm{~Hz}), 3.97(\mathrm{~s}, 3 \mathrm{H}), 4.17(\mathrm{q}, 1 \mathrm{H}, J=6.0 \mathrm{~Hz}), 4.58-4.59(\mathrm{~m}, 2 \mathrm{H}), 5.10(\mathrm{dd}$, $1 \mathrm{H}, J=2.0,11.6 \mathrm{~Hz}), 5.21(\mathrm{~s}, 1 \mathrm{H}), 5.31(\mathrm{dd}, 1 \mathrm{H}, J=0.8,10.8 \mathrm{~Hz}), 5.51(\mathrm{dd}$, $1 \mathrm{H}, J=0.8,17.2 \mathrm{~Hz}), 6.06(\mathrm{ddt}, 1 \mathrm{H}, J=10.8,17.2,4.8 \mathrm{~Hz}), 6.49(\mathrm{~d}, 1 \mathrm{H}$, $J=8.8 \mathrm{~Hz}), 6.74(\mathrm{~s}, 1 \mathrm{H}, \mathrm{NH}), 7.48-7.52(\mathrm{~m}, 2 \mathrm{H}), 7.61-7.65(\mathrm{~m}, 1 \mathrm{H}), 7.62(\mathrm{~d}$ $1 \mathrm{H}, J=8.8 \mathrm{~Hz}), 8.13-8.15(\mathrm{~m}, 2 \mathrm{H}), 11.8(\mathrm{~s}, 1 \mathrm{H}, \mathrm{OH}) ;{ }^{13} \mathrm{C}$ NMR $\left(\mathrm{CDCl}_{3}\right.$, $125 \mathrm{MHz}$ ): 18.1, 20.6, 37.7, 52.5, 57.1, 69.5, 69.6, 70.8, 103.2, 103.7 (q, $\left.J_{\mathrm{CF}}=271 \mathrm{~Hz}\right), 113.8,116.9,121.9,128.6,128.9,129.8,132.1,132.5,133.7$, $156.0\left(\mathrm{q}, J_{\mathrm{CF}}=36 \mathrm{~Hz}\right), 159.0,159.3,167.3,171.5$; IR (neat): 3340, 2980, 2950, $1720,1650,1615,1550 \mathrm{~cm}^{-1}$. Anal. calcd for $\mathrm{C}_{27} \mathrm{H}_{28} \mathrm{~F}_{3} \mathrm{NO}_{8}$ : C, 58.80; H, 5.12; $\mathrm{N}, 2.54$. Found: C, 58.59; H, 4.89; N, 2.24 .

\section{Methyl 2-hydroxy-3-(4-O-benzyl-2,3,6-trideoxy-3-C-methyl-3- trifluoroacetamido- $\beta$-L-lyxo-hexopyranosyl)-6-(prop-2-ene-1- yloxy)benzoate (19)}

Colorless oil, 88\%; $R_{f} 0.60$ (hexane/EtOAc $\left.=7 / 3\right) ;[\alpha]_{\mathrm{D}}^{25}-72\left(c 1.3, \mathrm{CHCl}_{3}\right) ;{ }^{1} \mathrm{H}$ NMR $\left(\mathrm{CDCl}_{3}, 400 \mathrm{MHz}\right): 1.40(\mathrm{~d}, 3 \mathrm{H}, J=6.4 \mathrm{~Hz}), 1.77(\mathrm{dd}, 1 \mathrm{H}, J=11.6$, $12.4 \mathrm{~Hz}), 1.79(\mathrm{~s}, 3 \mathrm{H}), 2.13(\mathrm{dd}, 1 \mathrm{H}, J=2.0,12.4 \mathrm{~Hz}), 3.58(\mathrm{~s}, 1 \mathrm{H}), 3.95(\mathrm{~s}$ $3 \mathrm{H}), 4.00(\mathrm{q}, 1 \mathrm{H}, J=6.4 \mathrm{~Hz}), 4.53-4.55(\mathrm{~m}, 2 \mathrm{H}), 4.56(\mathrm{~d}, 1 \mathrm{H}, J=11.6 \mathrm{~Hz})$, $4.82(\mathrm{~d}, 1 \mathrm{H}, J=11.6 \mathrm{~Hz}), 4.94(\mathrm{dd}, 1 \mathrm{H}, J=2.0,11.6 \mathrm{~Hz}), 5.29(\mathrm{dd}, 1 \mathrm{H}, J=1.6$, $10.8 \mathrm{~Hz}), 5.49(\mathrm{dd}, 1 \mathrm{H}, J=1.6,17.2 \mathrm{~Hz}), 6.03(\mathrm{ddt}, 1 \mathrm{H}, J=10.8,17.2,4.4 \mathrm{~Hz})$ $6.37(\mathrm{~s}, 1 \mathrm{H}, \mathrm{NH}), 6.42(\mathrm{~d}, 1 \mathrm{H}, J=8.8 \mathrm{~Hz}), 7.30-7.40(\mathrm{~m}, 5 \mathrm{H}), 7.54(\mathrm{~d}, 1 \mathrm{H}$, $J=8.8 \mathrm{~Hz}), 11.60(\mathrm{~s}, 1 \mathrm{H}, \mathrm{OH}) ;{ }^{13} \mathrm{C} \mathrm{NMR}\left(\mathrm{CDCl}_{3}, 100 \mathrm{MHz}\right): 18.5,20.6,38.5$, $52.4,56.9,69.4,69.4,72.1,75.9,79.6,103.3,103.5,115.4\left(\mathrm{q}, J_{\mathrm{CF}}=287 \mathrm{~Hz}\right)$, $116.8,122.0,127.6,127.9,128.5,132.1,132.5,137.5,156.2\left(\mathrm{q}, J_{\mathrm{CF}}=35 \mathrm{~Hz}\right)$, 158.7, 159.0, 171.3; IR (neat): 3330, 2920, 1718, 1648, 1616, 1436, 1352, 1298,
1198, 1156, $1080 \mathrm{~cm}^{-1}$. Anal. calcd for $\mathrm{C}_{27} \mathrm{H}_{30} \mathrm{~F}_{3} \mathrm{NO}_{7}$ : C, 60.33; H, 5.63; N, 2.61. Found: C, $60.12 ; \mathrm{H}, 5.38 ; \mathrm{N}, 2.39$.

\section{Methyl 2-hydroxy-3-(4-O-benzyl-2,3,6-trideoxy-3- $C$-methyl-3-} azido- $\beta$-L-lyxo-hexopyranosyl)-6-(prop-2-ene-1-yloxy)benzoate (20) Colorless oil, 48\%; $R_{f} 0.51$ (hexane/EtOAc $\left.=4 / 1\right) ;[\alpha]_{\mathrm{D}}^{20}-76\left(c 1.15, \mathrm{CHCl}_{3}\right)$; ${ }^{1} \mathrm{H}$ NMR $\left(\mathrm{CDCl}_{3}, 500 \mathrm{MHz}\right): 1.23(\mathrm{~d}, 3 \mathrm{H}, J=6.3 \mathrm{~Hz}), 1.45(\mathrm{~s}, 3 \mathrm{H}), 1.88$ (brd, $1 \mathrm{H}, J=12.6 \mathrm{~Hz}), 2.12(\mathrm{dd}, 1 \mathrm{H}, J=11.5,12.6 \mathrm{~Hz}), 3.08(\mathrm{~s}, 1 \mathrm{H}), 3.84(\mathrm{q}$ $1 \mathrm{H}, J=6.3 \mathrm{~Hz}), 3.94(\mathrm{~s}, 3 \mathrm{H}), 4.53-4.55(\mathrm{~m}, 2 \mathrm{H}), 4.61(\mathrm{~d}, 1 \mathrm{H}, J=10.9 \mathrm{~Hz}), 4.90$ (brd, $1 \mathrm{H}, J=11.5 \mathrm{~Hz}), 4.96(\mathrm{~d}, 1 \mathrm{H}, J=10.9 \mathrm{~Hz}), 5.28(\mathrm{dd}, 1 \mathrm{H}, J=1.2$ $10.9 \mathrm{~Hz}), 5.49(\mathrm{dd}, 1 \mathrm{H}, J=1.2,17.2 \mathrm{~Hz}), 6.02(\mathrm{ddt}, 1 \mathrm{H}, J=10.9,17.2$ $5.8 \mathrm{~Hz}), 6.43(\mathrm{~d}, 1 \mathrm{H}, J=8.6 \mathrm{~Hz}), 7.26-7.46(\mathrm{~m}, 5 \mathrm{H}), 7.58(\mathrm{~d}, 1 \mathrm{H}, J=8.6 \mathrm{~Hz})$ $11.66(\mathrm{~s}, 1 \mathrm{H}, \mathrm{OH}) ;{ }^{13} \mathrm{C} \mathrm{NMR}\left(\mathrm{CDCl}_{3}, 125 \mathrm{MHz}\right): 17.9,22.5,36.6,52.3,62.8$, 69.4, 69.8, 72.4, 75.6, 81.8, 103.2, 103.7, 116.8, 122.3, 127.6, 128.2, 128.3, 132.5, 132.7, 138.1, 158.8, 159.1, 171.6; IR (neat): 2891, 2101, 1736, 1654, 1618, 1439 1357, 1301, 1259, 1206, 1151, 1084, 984, 812, 755, 697, 664, $548 \mathrm{~cm}^{-1}$. Anal. calcd for $\mathrm{C}_{25} \mathrm{H}_{29} \mathrm{~N}_{3} \mathrm{O}_{6}$ : C, 64.23; $\mathrm{H}, 6.25 ; \mathrm{N}, 8.99$. Found: $\mathrm{C}, 64.07 ; \mathrm{H}, 6.38 ; \mathrm{N}$, 9.25 .

\section{Methyl 4-O-benzyl-2,3,6-trideoxy-3-C-methyl-3-dimethylamino-} $\beta$-L-lyxo-hexopyranoside (21)

To a solution of amine $13(76.0 \mathrm{mg}, 0.29 \mathrm{mmol})$ in $\mathrm{MeCN}(4 \mathrm{ml})$, formalin $(37 \%, 0.64 \mathrm{ml}, 8.6 \mathrm{mmol})$ and $\mathrm{NaBH}_{3} \mathrm{CN}(138 \mathrm{mg}, 2.19 \mathrm{mmol})$ were added at $0{ }^{\circ} \mathrm{C}$. After stirring for $15 \mathrm{~min}$, the reaction was quenched with $2 \mathrm{M}$ aqueous $\mathrm{NaOH}$. After extraction $\left(\mathrm{CH}_{2} \mathrm{Cl}_{2}, 6 \times\right)$, the combined organic extracts were successively washed with water and brine, and then dried $\left(\mathrm{Na}_{2} \mathrm{SO}_{4}\right)$. Filtration and concentration in vacuo gave dimethylamine $21(82.4 \mathrm{mg}, 98 \%)$ as a colorless oil. An analytical sample of $\mathbf{1 2}$ was obtained by preparative TLC (hexane/EtOAc/NEt $3=6 / 4 / 0.5$ ) as a colorless oil.

$\mathrm{R}_{f} 0.65$ (hexane/EtOAc/NEt $\left.{ }_{3}=6 / 4 / 1\right) ;[\alpha]_{\mathrm{D}}^{28}+44\left(c 0.91, \mathrm{CHCl}_{3}\right) ;{ }^{1} \mathrm{H} \mathrm{NMR}$ $\left(\mathrm{CDCl}_{3}, 500 \mathrm{MHz}\right): 0.98(\mathrm{~s}, 3 \mathrm{H}), 1.25(\mathrm{~d}, 3 \mathrm{H}, J=6.3 \mathrm{~Hz}), 1.65(\mathrm{dd}, 1 \mathrm{H}, J=2.3$ $11.5 \mathrm{~Hz}), 1.91(\mathrm{dd}, 1 \mathrm{H}, J=9.8,11.5 \mathrm{~Hz}), 2.25(\mathrm{~s}, 6 \mathrm{H}), 3.11(\mathrm{~s}, 1 \mathrm{H}), 3.50(\mathrm{~s}$ $3 \mathrm{H}), 3.66(\mathrm{q}, 1 \mathrm{H}, J=6.3 \mathrm{~Hz}), 4.51(\mathrm{dd}, 1 \mathrm{H}, J=2.3,9.8 \mathrm{~Hz}), 4.64(\mathrm{~d}, 1 \mathrm{H}$ $J=11.5 \mathrm{~Hz}), 4.96(\mathrm{~d}, 1 \mathrm{H}, J=11.5 \mathrm{~Hz}), 7.23-7.32(\mathrm{~m}, 3 \mathrm{H}), 7.41-7.46(\mathrm{~m}, 2 \mathrm{H})$ ${ }^{13} \mathrm{C}$ NMR $\left(\mathrm{CDCl}_{3}, 125 \mathrm{MHz}\right): 14.0,18.1,37.1,37.8,56.3,59.4,70.0,74.4,78.7$ 100.7, 127.1, 127.8, 128, 139.3; IR (neat): 2982, 2882, 1453, 1391, 1335, 1256, $1207,1131,1072,1014,965,884,736,700,592 \mathrm{~cm}^{-1}$. Anal. calcd for $\mathrm{C}_{17} \mathrm{H}_{27} \mathrm{NO}_{3}$ : C, 69.59; H, 9.28; N, 4.77. Found: C, 69.49; H, 9.15; N, 4.57.

One-pot conversion of azide 14 to dimethylamine 21. To a solution of azide 14 $(25.3 \mathrm{mg}, 86.8 \mu \mathrm{mol})$ in $\mathrm{CH}_{2} \mathrm{Cl}_{2}(1.7 \mathrm{ml}), \mathrm{PMe}_{3}(1 \mathrm{M}$ in toluene, $0.26 \mathrm{ml}$ $0.26 \mathrm{mmol}$ ) was added at room temperature. After stirring for $4 \mathrm{~h}$, the solvent was removed in vacuo, and the residue was dissolved in $\mathrm{MeCN}(1.7 \mathrm{ml})$ Formalin $(37 \%, 210 \mu \mathrm{l}, 2.8 \mathrm{mmol})$ was added at room temperature, and the mixture was stirred for $30 \mathrm{~min}$. The $\mathrm{pH}$ was adjusted to $\mathrm{pH} 4$ with $\mathrm{AcOH}$ and $\mathrm{NaBH}_{3} \mathrm{CN}(27 \mathrm{mg}, 0.43 \mathrm{mmol})$ was added at room temperature. After stirring for $1 \mathrm{~h}$, the reaction was quenched with saturated aqueous $\mathrm{NaHCO}_{3}$ at $0{ }^{\circ} \mathrm{C}$ After extraction with EtOAc $(3 \times)$, the combined organic extracts were washed with brine and then dried $\left(\mathrm{Na}_{2} \mathrm{SO}_{4}\right)$. After filtration, solvents were removed in vacuo, and the residue was purified by preparative TLC (hexane/EtOAc/ $\left.\mathrm{NEt}_{3}=6 / 4 / 0.5\right)$ to give dimethylamine $21(18.9 \mathrm{mg}, 74 \%)$ as a colorless oil.

\section{ACKNOWLEDGEMENTS}

This work was supported by Grant-in-Aid for Specially Promoted Research (No. 23000006) from JSPS, Japan. We are grateful to Dr Hidehiro Uekusa and Ms Sachiyo Kubo for X-ray analysis.

1 Johnson, A. W., Smith, R. M. \& Guthrie, R. D. Vancosamine: the structure and configuration of a novel amino-sugar from vancomycin. J. Chem. Soc. Perkin Trans. 1, 2153-2159 (1972).

2 Weringa, W. D., Williams, D. H., Feeney, J., Brown, J. P. \& King, R. W. The structure of an amino-sugar from the antibiotic vancomycin. J. Chem. Soc. Perkin Trans. 1, 443-446 (1972)

3 Williams, D. H. \& Bardsley, B. The vancomycin group of antibiotics and the fight against resistant bacteria. Angew. Chem. Int. Ed. 38, 1172-1193 (1999). 
4 Tanaka, Y., Gräfe, U., Yazawa, K., Mikami, Y. \& Ritzau, M. Nocardicyclins A and B: new anthracycline antibiotics produced by Nocardia pseudobrasiliensis. J. Antibiot. 50, 822-827 (1997).

5 Tanaka, Y., Gräfe, U., Yazawa, K. \& Mikami, Y. Production of nocardicyclins by clinical isolates of Nocardia pseudobrasiliensis and in vivo antitumor activity of the antibiotic. J. Antibiot. 51, 589-591 (1998).

6 Maeda, K. et al. A new antitumor substance, pluramycin. J. Antibiot. 9, 75-81 (1956).

7 Kondo, S, Miyamoto, M., Naganawa, H., Takeuchi, T. \& Umezawa, H. Structures of pluramycin A and neopluramycin. J. Antibiot. 30, 1143-1145 (1977).

8 Bililign, T., Griffith, B. R. \& Thorson, J. S. Structure, activity, synthesis and biosynthesis of aryl C-glycosides. Nat. Prod. Rep. 22, 742-760 (2005).

9 Thang, T. T., Winternitz, F., Olesker, A., Lagrange, A. \& Lukacs, G. Synthesis of a derivative of vancosamine, a component of the glycopeptide antibiotic vancomycin. J. Chem. Soc. Chem. Commun. 153-154 (1979).

10 Thang, T. T., Winternitz, F., Lagrange, A., Olesker, A. \& Lukacs, G. Stereospecific access to branched-chain carbohydrate synthons. Tetrahedron Lett. 21, 4495-4498 (1980).

11 Ahmad, H. I., Brimacombe, J. S., Mengech, A. S. \& Tucker, L. C. N. The synthesis of some derivatives of L-vancosamine (3-amino-2,3,6-trideoxy-3-C-methyl-L-lyxo-hexose). Carbohydr. Res. 93, 288-293 (1981).

12 Brimacombe, J. S., Mengech, A. S., Rahman, K. M. M. \& Tucker, L. C. N. An approach to branched-chain amino sugars, particulary derivatives of L-vancosamine (3-amino2,3,6-trideoxy-3-C-methyl-L-lyxo-hexose) and its $D$ enantiomer, via the cyano-hydrin route. Carbohydr. Res. 110, 207-215 (1982).

13 Dyong, I., Weigand, J. \& Thiem, J. Synthesen ungesättigter Aminozucker und Aminoalkyl-verzweigter Kohlenhydrate durch sigmatrope Umlargerung von Trichloracetimidaten. Liebigs Ann. Chem. 577-599 (1986).

14 Klemer, A. \& Wilbers, H. Neue Synthesen von Derivaten der Antibiotikazucker 3 Amino-2,3,6-tridesoxy-3-C-methyl-L-xylo-hexopyranose, L-Vancosamine, D-Rubranitrose und von Vorläufen der L-Decilonitrose und D-Kijanose. Liebigs Ann. Chem. 815-823 (1987).

15 Greven, R., Jütten, P. \& Scharf, H.-D. Stereoselective synthesis of L-vancosamine methyl $\beta$-glycoside by addition of an organocerium reagent to $O$-benzyloxime ethers. Carbohydr. Res. 275, 83-93 (1995).

16 Smith, G. R. \& Giuliano, R. M. Synthesis of methyl $\alpha$-L-vancosaminide. Carbohydr. Res. 323, 208-212 (2000).

17 Dyong, I. \& Friege, H. N-Acetyl-1,4-di-O-acetyl- $\beta$-DL-vancosamin. Chem. Ber. 112, 3273-3281 (1979).

18 Dyong, I., Friege, H., Luftmann, H. \& Merten, H. Totalsynthese und Konfigurationsbestimmung Me-C(3)-NHR-verzweigter 2,3,6-tridesoxyhexosen. Chem. Ber. 114, 2669-2680 (1981).

19 Fronza, G., Fuganti, C., Grasselli, P. \& Pedrocchi-Fantoni, G. Synthesis of the $\mathrm{N}$-benzoyl derivatives of L-arabino, L-lyxo (L-vancosamine) isomers of 2,3,6-trideoxy3-C-methyl-3-aminohexose from a non-carbohydrate precursor. Tetrahedron Lett. 22 , 5073-5076 (1981)

20 Fronza, G., Fuganti, C., Grasselli, P. \& Pedrocchi-Fantoni, G. Synthesis of the four configuratonal isomers of $\mathrm{N}$-benzyl-2,3,6-trideoxy-3-C-methyl-3-amino-L-hexose from the $(2 S, 3 R)$-diol obtained from $\alpha$-methylcinnamaldehyde by fermentation with Baker's yeast. J. Carbohydr. Chem. 2, 225-248 (1983).

21 Hamada, Y., Kawai, A. \& Shioiri, T. New methods and reagents in organic synthesis. 50. A stereoselective synthesis of a derivative of L-vancosamine, a carbohydrate component of the antibiotics vancomycin and sporaviridin. Tetrahedron Lett. 25, 5413-5414 (1984).

22 Hauser, F. M., Ellenberger, S. R., Glusker, J. P., Smart, C. J. \& Carrell, H. L. Stereoselective syntheses of $( \pm)$-daunosamine, $( \pm)$-vancosamine, and $( \pm)$-ristosamine from acyclic precursors. J. Org. Chem. 51, 50-57 (1986).

23 Hamada, Y., Kawai, A., Matsui, T., Hara, O. \& Shioiri, T. 4-Alkoxycarbonyloxazoles as $\beta$ hydroxy- $\alpha$-amino acid synthons: efficient, stereoselective synthesis of 3-amino-2,3,6trideoxyhexoses and a hydroxy amino acid moiety of Al-77-B. Tetrahedron 46, 4823-4846 (1990).
24 Nicolaou, K. C., Mitchell, H. J., van Delft, F. L., Rübsam, F. \& Rodríguez, R. M. Expeditious routes to evernitrose and vancosamine derivatives and synthesis of a model vancomycin aryl glycoside. Angew. Chem. Int. Ed. 37, 1871-1874 (1998).

25 Nicolaou, K. C. et al. Total synthesis of vancomycin-Part 4: attachment of the sugar moieties and completion of the synthesis. Chem. Eur. J. 5, 2648-2667 (1999).

26 Nicolaou, K. C., Baran, P. S., Zhong, Y.-L. \& Vega, J. A. Novel IBX-mediated processes for the synthesis of amino sugars and libraries thereof. Angew. Chem. Int. Ed. 39, 2525-2529 (2000).

27 Cutchins, W. W. \& McDonald, F. E. Stereoselective synthesis of vancosamine and saccharosamine glycals via tungsten-catalyzed alkynol cycloisomerization. Org. Lett. 4, 749-752 (2002).

28 Parker, K. A. \& Chang, W. A synthesis of L-vancosamine derivatives from noncarbohydrate precursors by a short sequence based on the Marshall, McDonald, and Du Bois reactions. Org. Lett. 5, 3891-3893 (2003).

29 Thang, T. T. et al. Synthesis and antitumor activity of 3'-C-methyl-daunorubicin. Carbohydr. Res. 135, 241-247 (1985).

$30 \mathrm{Hsu}$, D.-S., Matsumoto, T. \& Suzuki, K. Synthesis of L-vancosamine derivatives from methyl $\alpha$-D-mannopyranoside. Synlett 469-471 (2006).

31 Horton, D. \& Weckerle, W. A preparative synthesis of 3-amino-2,3,6-trideoxy-L-lyxohexose (daunosamine) hydrochloride from D-mannose. Carbohydr. Res. 44, 227 (1975).

32 Imamoto, T. et al. Carbon-carbon bond forming reactions using cerium metal or organocerium(III) reagents. J. Org. Chem. 49, 3904-3912 (1984).

33 Takeda, N. \& Imamoto, T. Use of cerium(III) chloride in the reactions of carbonyl compounds with organolithium or Grignard reagents for the suppression of abnormal reactions: 1-butyl 1,2,3,4-tetrahydro-1-naphthol. Org. Synth. 76, 228-233 (1999).

34 Keck, G. E., McHardy, S. F. \& Wager, T. T. Reductive cleavage of the $\mathrm{N}-0$ bonds in hydroxylamine and hydroxamic acid derivatives using $\mathrm{Sml}_{2}$ /THF. Tetrahedron Lett. 36, 7419-7422 (1995).

35 Imamoto, T. \& Ono, M. The reaction of samarium(III) iodide with samarium metal in tetrahydrofuran. A new method for the preparation of samarium(II) iodide. Chem. Lett. 501-502 (1987).

36 Hanessian, S. The reaction of $\mathrm{O}$-benzylidene sugars with $\mathrm{N}$-bromosuccinimide I. Methyl 4,6-0-benzylidenehexopyranosides. Carbohydr. Res. 2, 86-88 (1966).

37 Hanessian, S. \& Plessas, N. R. The reaction of O-benzylidene sugars with $\mathrm{N}$-bromosuccinimide. II. Scope and synthetic utility in the methyl 4,6-O-benzylidenehexopyranoside series. J. Org. Chem. 34, 1035-1044 (1969).

38 Duhamel, L., Plé, G., Angibaud, P. \& Desmurs, J. R. New chiral brominating and chlorinating agents. Synth. Commun. 23, 2423-2433 (1993).

39 Wang, L.-X., Sakairi, N \& Kuzuhara, H. Synthesis of 1D-(1,3,5/2,4)-4-acetamido-5amino-1,2,3-cyclohexanetriol and its incorporation into a pseudo-disaccharide. Carbohydr. Res. 275, 33-47 (1995).

40 Lee, Y. J., Kubota, A., Ishiwata, A. \& Ito, Y. Synthesis of pseudaminic acid, a unique nonulopyranoside derived from pathogenic bacteria through 6-deoxy-AltdiNAc. Tetrahedron Lett. 52, 418-421 (2011).

41 Xu, Y.-C., Bizuneh, A. \& Walker, C. Selective deprotection of alkyl esters using magnesium methoxide. Tetrahedron Lett. 37, 455-458 (1996).

42 Kimura, Y., Matsumoto, T., Suzuki, M. \& Terashima, S. An improved synthesis of $N$ trifluoroacetyl-L-daunosamine. Bull. Chem. Soc. Jpn 59, 663-664 (1986).

43 Alper, P. B., Hung, S.-C. \& Wong, C.-H. Metal catalyzed diazo transfer for the synthesis of azides from amines. Tetrahedron Lett. 37, 6029-6032 (1996).

44 Titz, A., Radic, Z., Schwardt, O. \& Ernst, B. A safe and convenient method for the preparation of triflyl azide, and its use in diazo transfer reactions to primary amines. Tetrahedron Lett. 47, 2383-2385 (2006).

45 Kitamura, M. et al. Direct synthesis of organic azides from primary amines with 2-azido1,3-dimethylimidazolinium hexafluorophosphate. Eur. J. Org. Chem. 458-462 (2011).

46 Kato, H., Ohmori, K. \& Suzuki, K. Convenient procedure for one-pot conversion of azides to $\mathrm{N}$-monomethylamines. Synlett 1003-1005 (2001).

47 Ben, A., Hsu, D.-S., Matsumoto, T. \& Suzuki, K. Total synthesis and structure revision of deacetylravidomycin M. Tetrahedron 67, 6460-6468 (2011). 